https://helda.helsinki.fi

\title{
Proof-Theoretic Analysis of the Quantified Argument Calculus
}

\section{pÿPavlovi , Edi}

\section{9-12}

pÿPavlovi , E \& Norbert Gratzl 2019 , ' Proof-Theoretic Analysis of the Quantified Argument

Calculus ', The Review of Symbolic Logic , vol. 12 , no. 4 , 1755020318000114 , pp.

607-636 . https://doi.org/10.1017/S1755020318000114

http://hdl.handle.net/10138/305478

https://doi.org/10.1017/S1755020318000114

cc_by_nc_nd

acceptedVersion

Downloaded from Helda, University of Helsinki institutional repository.

This is an electronic reprint of the original article.

This reprint may differ from the original in pagination and typographic detail.

Please cite the original version. 
DOI:10.1017/S1755020318000114

This is a manuscript accepted for publication in The Review of Symbolic Logic. Changes are likely to be introduced during the production process.

\title{
Proof-Theoretic Analysis of The Quantified Argument Calculus
}

\author{
Edi Pavlović, Norbert Gratzl ${ }^{\dagger}$
}

\begin{abstract}
This paper investigates the proof theory of the Quantified Argument Calculus (Quarc) as developed and systematically studied by Hanoch BenYami [3], [4]. Ben-Yami makes use of natural deduction (Suppes-Lemmonstyle), we, however, have chosen a sequent calculus presentation, which allows for the proofs of a multitude of significant meta-theoretic results with minor modifications to the Gentzen's original framework, i.e. LK. As will be made clear in course of the paper LK-Quarc will enjoy cut elimination and its corollaries (including subformula property and thus consistency).
\end{abstract}

\section{Introduction}

This paper investigates the proof theory of the Quantified Argument Calculus (Quarc) as developed and systematically studied by Hanoch Ben-Yami [3], [4]. Ben-Yami makes use of natural deduction (Suppes-Lemmon-style), we, however, have chosen a sequent calculus presentation, which allows for the proofs of a multitude of significant meta-theoretic results with minor modifications to the Gentzen's original framework, i.e. LK. LK, although it has been developed in the 1930ies serves still (as a basis) for proof theoretic investigations [1], [6], [15].

Quarc is a system of quantified logic which does away with variables and unrestricted predicates, but nonetheless achieves results similar to the Predicate Calculus by employing quantifiers applied directly to predicates which appear as arguments of other predicates (hence the name Quantified Argument Calculus), along with anaphors and operators that attach directly to predicates. It is in this respect arguably closer to natural language. ${ }^{1}$ A goal of this paper is to show how and to what extent some of these results are achieved. Given that this is an interesting but not widely known system, we will present it here in considerable detail before proceeding with the proof-theoretic analysis of it. The

\footnotetext{
*This paper was finalized in part due to the support of the Academy of Finland, research project no. 1308664 .

${ }^{\dagger}$ This work was supported by the Marie-Sklodowska-Curie Innovative Training Network DIAPHORA.

${ }^{1}$ We thank an anonymous referee for pointing this out.
} 
reason to use sequent calculus in this analysis is to provide a constructive proof of consistency, but first and foremost to prove an important, useful and interesting result of a cut elimination theorem and its corollaries. We are likewise able to straightforwardly extend the system with the identity, which does not appear in [3].

The way the research on Quarc is conducted here is as follows: we observe first that Ben-Yami's Quarc is a rather rich system. In our analysis we split up Quarc into three distinct sub-systems, namely (1) LK-Quarc ${ }_{B}$, (2) LK-Quarc 2 , (3) LK-Quarc 3 , and finally, LK-Quarc - which is Ben-Yami's (full) Quarc. LKQuarc $_{B}$ does not contain either the rules for identity or instantiation. LKQuarc $_{2}$ is an extension of LK-Quarc ${ }_{B}$ with identity, and LK-Quarc 3 an extension of LK-Quarc ${ }_{B}$ with the rule for instantiation. Finally, LK-Quarc is obtained by combining LK-Quarc 2 and LK-Quarc 3 . As will be made clear in course of the paper LK-Quarc will enjoy cut elimination and its corollaries (including subformula property and thus consistency which is not proven in [3], although it follows almost immediately from the soundness proof present there).

Let us note that the quantifiers in Quarc do have particular import, a fact that is expressed semantically by the condition of non-emptiness of (unary) predicates. This is in contrast to first-order predicate logic, where, as it is well known, (unary) predicates can be empty. On the level of theorems we make distinctions on the strength of particular import. On the basic level, i.e. LKQuarc $_{B}$, this is expressed by the following formulas (the notation of Quarc and its language will be explained in some detail in section 2 of this paper): (1) $(\forall S) P \rightarrow((\exists S) S \rightarrow(\exists S) P)$ - example: if all men are mortal, then if there are men, then some men are mortal; and $(2)(\forall S) P \rightarrow(a S \rightarrow a P)$, e.g. if all men are mortal, then if Socrates is a man, then Socrates is mortal. The strong version of particular import, that is, $(3)(\forall S) P \rightarrow(\exists S) P$ is a theorem of LK-Quarc 3 . Clearly, $(\exists S) S$, which can be read as "there are $\mathrm{S}$ ", is a theorem of Quarc 3 as well. However, this is not to be conflated with the existential construction "S exist", as noted by Ben-Yami in [3] and discussed in more detail in [4]. Following that, the quantifier $\exists$ is referred to as particular quantifier in this paper.

Focusing for the moment on formula (2), there is a striking similarity with quantification in free logic [10], [11] and its most distinct axiom: $\forall x A \rightarrow(E ! a \rightarrow$ $A[a / x])$. Of course, in free logic (as in first-order logic), predicates can be empty, but still there is a structural parallel. As a matter of fact, this parallel will be exploited in the corresponding formulations of the sequent rules related to the quantifiers; more on this similarity is said below (p.11) of this paper.

A note on some of the other special symbols - Quarc introduces additional logical symbols and operations of Anaphora, Reorder and Negative Predication. Anaphora fulfills a role roughly similar, but broader, than that of the variables in Predicate calculus and is crucial in determining which parts of the formula a quantified argument governs. Reorder is an operation that replaces predicates with those which contain arguments in different order. Reordered predicates 
are interchangeable with identity-permutation ones in the basic case, but not in the quantified case, and are used to determine mutual governance in a multiply quantified formula. Negative Predication is an operation that switches between sentential negation (e.g. $\neg(a) S$, it is not the case that a is $\mathrm{S}$ ) and predication negation (e.g. $(a) \neg S$, a isn't $S$ ). Again, these two uses of negation are interchangeable in the basic, but not the quantified case (compare: $\neg(\exists S) P$, it is not the case that some $\mathrm{S}$ are $\mathrm{P}$ and $(\exists S) \neg P$, some $\mathrm{S}$ aren't $\mathrm{P})$, and are therefore used to determine the mutual scope of negations and quantifiers. Most of the proofs in this paper will focus on the quantifiers and the related additional special symbols, as those are the primary novelty of Quarc.

Plan of the paper: In section 2 we present Quarc $_{B}$, consisting of its language, truth-value assignments and derivation rules (natural deduction - following BenYami) with appropriate modifications for the purposes of this paper. Section 3 sets out with the sequent calculus formulation of $\operatorname{Quarc}_{B}$. Section 4 proves the deductive equivalence of the two formulations of Quarc $_{B}$. The central section of this paper, 5, prove the cut elimination theorem and its corollaries (subformula property and consistency) for LK-Quarc ${ }_{B}$. Section 6 expands LK-Quarc ${ }_{B}$ with the rules for identity, proves again deductive equivalence, cut elimination and its corollaries and furthermore conservativity over LK-Quarc ${ }_{B}$. Section 7 extends LK-Quarc ${ }_{B}$ with a rule of instantiation and once again proves all the results from above for LK-Quarc 3 .

\section{$2 \operatorname{Quarc}_{B}$}

The system presented here will be Quarc $_{B}$, which differs from the full Quarc in containing no rules for identity and instantiation.

\section{$2.1 \quad$ Vocabulary}

Definition 1 (Vocabulary of $\operatorname{Quarc}_{B}$ ) Quarc $B$ contains the following symbols:

1. Predicates: $P, Q, R, \ldots$, denumerably many and with a fixed arity.

2. Reordered predicates: For every $n$-ary $(n>1)$ predicate $R$, reordered predicates $R^{\pi}$, where $\pi$ is any permutation of $1, \ldots, n$ except identity permutation.

3. Singular arguments (SA's): $a, b, c, \ldots$

4. Anaphors: $\alpha, \beta, \gamma, \ldots$

5. Sentential operators: $\neg, \vee, \wedge, \rightarrow$.

6. Quantifiers: $\forall, \exists$.

7. Numerals used as indices, comma, parentheses. 


\subsection{Formula}

A note on notation - we use a (metalinguistic) notation $A[\varphi]$ to describe a formula $A$ which contains the strings of symbols $\varphi$, and $A[\psi / \varphi]$ to describe a formula $A$ where the string of symbols $\varphi$ is substituted by a string of symbols $\psi$.

Definition 2 (Formula) The following rules specify all the ways in which formulas can be generated.

1. (Basic formula) If $P$ is an $n$-ary predicate and $t_{1}, \ldots, t_{n}$ SA's, then $\left(t_{1}, \ldots, t_{n}\right) P$ is a formula, called a basic formula.

2. (Reorder) If $P$ is a reordered $n$-ary predicate $(n>1)$ and $t_{1}, \ldots, t_{n}$ SA's, then $\left(t_{1}, \ldots, t_{n}\right) P$ is a formula. Note that reordered predicates are a separate class of symbols of the language, and so no formula containing a reordered predicate is basic.

3. (Negative predication) If $P$ is an $n$-ary predicate or a reordered $n$-ary predicate and $t_{1}, \ldots, t_{n} \mathrm{SA}$ 's, then $\left(t_{1}, \ldots, t_{n}\right) \neg P$ is a formula.

4. (Sentential operators) If $A$ and $B$ are formulas, so are $\neg(A),(A) \wedge(B),(A) \vee$ $(B),(A) \rightarrow(B)$. The parentheses surrounding formulas are called sentential parentheses.

5. (Anaphora) If $A$ is a formula containing, from left to right, $t_{1}, \ldots, t_{n}(n>1)$ occurrences of SA $t$, none of which is a source of any anaphora, and $A$ does not contain $\alpha$, then $A\left[t_{\alpha} / t_{1}, \alpha / t_{2}, \ldots, \alpha / t_{n}\right]$ is a formula. We call $t_{\alpha}$ the source of the anaphora $\alpha$.

6. (Quantification) If $P$ is a unary predicate, then $\forall P$ and $\exists P$ are quantified arguments (QA's). If $A$ is a formula containing an occurrence of an SA $t$, and substituting a QA $q P$ for $t$ will result in $q P$ governing $A$, then $A[q P / t]$ is a formula.

Obviously, to make sense of the last entry, we also need to define governance:

7. (Governance) An occurrence $q P$ of a $\mathrm{QA}$ governs a formula $A$ just in case $q P$ is the leftmost QA in $A$ and $A$ does not contain any other string of symbols $(B)$ in which the parentheses are a pair of sentential parentheses, such that $B$ contains $q P$ and all the anaphors of all the QA's in $B$.

Closer inspection of the rules shows that some of these can be applied in multiple orders. Namely, applications of the anaphora rule can be transposed with one or more applications of the quantifier, sentential operator, or anaphora rules. Whenever such a situation occurs, as a matter of convention, anaphora rules are applied first. Among the anaphora rules, first applied is that which has 
then rightmost argument as its source. Given that every anaphor has a single source, and no two anaphors have the same source, this convention produces a unique order of applications of formula-generation rules.

Definition 3 (Terminal Symbol) The symbol introduced, for any formula, by the last application of a formula-generation rule is called a terminal symbol of that formula.

\subsection{Truth-Value Assignments}

We now define the truth-value assignments for formulas of Quarc. The semantics given in [3] are substitutional, and, even though Quarc is not essentially substitutional (a model-theoretic approach was used for a similar system in [12]), we will not alter it, given that no result in this paper hinges on the distinction.

Definition 4 (Truth-Value Assignments) For any truth-value assignment $A$, the following holds:

1. (Basic formula) Every basic formula is assigned the truth-value of true or false, but not both.

2. (Reorder) Let $P$ be an $n$-ary predicate and $\pi=\pi_{1}, \ldots, \pi_{n}$ a permutation of $1, \ldots, n$. Then, the truth-value assigned to $\left(t_{\pi 1}, \ldots, t_{\pi n}\right) P^{\pi}$ is that assigned to $\left(t_{1}, \ldots, t_{n}\right) P$.

3. (Sentential operators) Let $A$ and $B$ be formulas. Then, $\neg(A)$ is true just in case $A$ is false. Etc.

4. (Negative predication) Let $P$ be an $n$-ary predicate and $t_{1}, \ldots, t_{n}$ SA's. The truth value of $\left(t_{1}, \ldots, t_{n}\right) \neg P$ is that of $\neg\left(t_{1}, \ldots, t_{n}\right) P$.

5. (Anaphora) If $A$ is a formula containing, from left to right, occurrences $t_{1}, \ldots, t_{n}$ of SA $t$, none of which is the source of any anaphors, and $A$ does not contain $\alpha$, then the truth-value of $A\left[t_{\alpha} / t_{1}, \alpha / t_{2}, \ldots, \alpha / t_{n}\right]$ is that of $A$.

6. (Quantification) Let $A[\forall P](A[\exists P])$ be formula $A$ governed by the QA $\forall P$ $(\exists P)$. If for every (some) SA $t$ for which $(t) P$ is true $A[t / \forall P](A[t / \exists P])$ is true, then $A$ is true, and false otherwise.

In addition to these, one of the rules needed for full Quarc is that of instantiation:

7. (Instantiation) For any unary predicate $P$ there is an SA $t$ such that $(t) P$ is true. 


\subsection{Derivation Rules}

The rules presented here are taken from [3]. We only present the rules specific to Quarc; the rules for propositional connectives are standard and will be omitted. We begin by a definition of a proof, due to [5]:

Definition 5 (Proof) A proof is a list of lines of the form $\langle L,(i), A, R\rangle$, where $L$ is a (possibly empty) sequence of formulas, $(i)$ the line number, $A$ a formula and $R$ a justification, an element of the set of the derivation rules.

Definition 6 (Derivation Rules) The following are the derivation rules of Quarc ${ }_{B}$ :

1. (Premise)

\section{i (i) $A$ Premise}

2. (Propositional Calculus) We allow the usual derivation rules of the Propositional Calculus, with the constraint that for each rule, the principal formulas be formulas of Quarc. E.g. one cannot obtain $(\alpha) P$ from $\left(t_{\alpha}\right) S \wedge$ $(\alpha) P$ by $\wedge$ E.

3. (Sentence to Predication Negation, SP)

$L \quad\left(\right.$ i) $\quad \neg\left(t_{1}, \ldots, t_{n}\right) P$

$L \quad\left(\right.$ j) $\quad\left(t_{1}, \ldots, t_{n}\right) \neg P \quad$ SP, i

4. (Predication to Sentence Negation, PS)

$L \quad\left(\right.$ i) $\quad\left(t_{1}, \ldots, t_{n}\right) \neg P$

$L \quad$ (j) $\neg\left(t_{1}, \ldots, t_{n}\right) P \quad$ PS, i

5. (Reorder, R) Where $\pi$ and $\rho$ are any permutation of $1, \ldots, n$ including the identity permutation,

$L \quad$ (i) $\quad\left(t_{\pi 1}, \ldots, t_{\pi n}\right) P^{\pi}$

$L \quad(\mathrm{j}) \quad\left(t_{\rho 1}, \ldots, t_{\rho n}\right) P^{\rho} \quad \mathrm{R}, \mathrm{i}$

6. (Anaphora Introduction, AI) Where $t_{1}, \ldots, t_{n}$ are $n, n>1$, occurrences of the same singular argument $t$, none of $t_{1}, \ldots, t_{n}$ is a source of an anaphor, and $\alpha$ does not occur in $A$,

$$
\begin{array}{lll}
L & \text { (i) } & A\left[t_{1}, \ldots, t_{n}\right] \\
L & \text { (j) } & A\left[t_{\alpha} / t_{1}, \alpha / t_{2} \ldots, \alpha / t_{n}\right] \quad \text { AI, i }
\end{array}
$$

7. (Anaphora Elimination, AE) Where same provisions as in AI apply,

$$
\begin{array}{lll}
L & \text { (i) } & A\left[t_{\alpha} / t_{1}, \alpha / t_{2} \ldots, \alpha / t_{n}\right] \\
L & \text { (j) } & A\left[t_{1}, \ldots, t_{n}\right]
\end{array} \quad \mathrm{AE}, \mathrm{i}
$$


8. (Universal Introduction, UI) Where $A[\forall P]$ is governed by the quantified argument $\forall P$, and the singular argument $t$ does not occur in any of the premises listed in $L$ apart from (i), nor in $A[\forall P]$,

$\begin{array}{rlll}i & (\mathrm{i}) & (t) P & \text { Premise } \\ L^{*} & (\mathrm{j}) & A[t / \forall P] & \\ L-\{i\} & (\mathrm{k}) & A[\forall P] & \mathrm{UI}, \mathrm{i}, \mathrm{j}\end{array}$

9. (Universal Elimination, UE) Where $A[\forall P]$ is governed by the quantified argument $\forall P$ as above,

$$
\begin{aligned}
& L_{1} \quad \text { (i) } \quad A[\forall P] \\
& L_{2} \quad(\mathrm{j}) \quad(t) P \\
& L_{1} \cup L_{2} \quad(\mathrm{k}) \quad A[t / \forall P] \quad \mathrm{UE}, \mathrm{i}, \mathrm{j}
\end{aligned}
$$

10. (Particular Introduction, PI) Where $A[\exists P]$ is governed by the quantified argument $\exists P$,

$$
\begin{array}{rrll}
L_{1} & (\mathrm{i}) & A[t / \exists P] \\
L_{2} & (\mathrm{j}) & (t) P \\
L_{1} \cup L_{2} & (\mathrm{k}) & A[\exists P] \quad \text { PI, i, j }
\end{array}
$$

11. (Particular Elimination, $\mathrm{PE}$ ) Where $A[\exists P]$ is governed by the quantified argument $\exists P$, and the singular argument $t$ does not occur anywhere in $L_{1} \cup L_{2}-\{j, k\}, A[\exists P]$ or $B$,

$$
\begin{array}{rcll}
L_{1} & (\mathrm{i}) & A[\exists P] & \\
j & (\mathrm{j}) & (t) P & \text { Premise } \\
k & (\mathrm{k}) & A[t / \exists P] & \text { Premise } \\
L_{2} & (\mathrm{l}) & B & \\
L_{1} \cup L_{2}-\{j, k\} & (\mathrm{m}) & B & \mathrm{PE}, \mathrm{i}, \mathrm{j}, \mathrm{k}, \mathrm{l}
\end{array}
$$

Note that PE is a rule of Quarc ${ }_{B}$, but not full Quarc, which uses the rule of Instantiation. This rule resembles $\mathrm{PE}$ but is defined for either quantifier. Let $q$ be either $\exists$ or $\forall$ :

12. (Instantiation, Ins) Where same provisions apply, mutatis mutandis, as in $\mathrm{PE}$,

$$
\begin{array}{rcll}
L_{1} & (\mathrm{i}) & A[q P] & \\
j & (\mathrm{j}) & (t) P & \text { Premise } \\
k & (\mathrm{k}) & A[t / q P] & \text { Premise } \\
L_{2} & (\mathrm{l}) & B & \\
L_{1} \cup L_{2}-\{j, k\} & (\mathrm{m}) & B & \text { Ins, } \mathrm{i}, \mathrm{j}, \mathrm{k}, \mathrm{l}
\end{array}
$$


Consequently, the following is a theorem of full Quarc, but not (as we will see) Quarc $_{B}$ :

Theorem 1 (Particular Import in Quarc): $(\forall M) P \vdash(\exists M) P$

Proof.

$\begin{array}{rlll}1 & (1) & (\forall M) P & \text { Premise } \\ 2 & (2) & (a) M & \text { Premise } \\ 3 & (3) & (a) P & \text { Premise } \\ 2,3 & (4) & (\exists M) P & \text { PI, 2, 3 } \\ 1 & (5) & (\exists M) P & \text { Ins, 1, 2, 3, 4 }\end{array}$

\subsubsection{Examples}

In this section we provide several examples of the uses of (full) Quarc, namely to prove the syllogism Barbara and several instances of the DeMorgan laws.

Example 1 Syllogism Barbara

$(\forall M) P,(\forall S) M \vdash(\forall S) P$

Proof.

$\begin{array}{rlll}1 & (1) & (\forall M) P & \text { Premise } \\ 2 & (2) & (\forall S) M & \text { Premise } \\ 3 & (3) & (a) S & \text { Premise } \\ 2,3 & (4) & (a) M & \text { UE, } 2,3 \\ 1,2,3 & (5) & (a) P & \text { UE, } 1,4 \\ 1,2 & (6) & (\forall S) P & \text { UI, } 3,5\end{array}$

Example 2 DeMorgan Laws

$(\exists M) P \vdash \neg(\forall M) \neg P$

Proof.

$\begin{array}{rcll}1 & (1) & (\exists M) P & \text { Premise } \\ 2 & (2) & (\forall M) \neg P & \text { Premise } \\ 3 & (3) & (a) M & \text { Premise } \\ 4 & (4) & (a) P & \text { Premise } \\ 2,3 & (5) & (a) \neg P & \text { UE, } 2,3 \\ 2,3 & (6) & \neg(a) P & \text { PS, } 5 \\ 3,4 & (7) & \neg(\forall M) \neg P & \neg \mathrm{I}, 2,4,6 \\ 1 & (8) & \neg(\forall M) \neg P & \text { PE, } 1,3,4,7\end{array}$


$\neg(\forall M) \neg P \vdash(\exists M) P$

Proof.

$\begin{array}{rcll}1 & (1) & \neg(\forall M) \neg P & \text { Premise } \\ 2 & (2) & \neg(\exists M) P & \text { Premise } \\ 3 & (3) & (a) M & \text { Premise } \\ 4 & (4) & (a) P & \text { Premise } \\ 3,4 & (5) & (\exists M) P & \text { PI, } 3,4 \\ 2,3 & (6) & \neg(a) P & \neg \mathrm{I}, 4,2,5 \\ 2,3 & (7) & (a) \neg P & \text { SP, } 6 \\ 2 & (8) & (\forall M) \neg P & \text { UI, } 3,7 \\ 1 & (9) & \neg \neg(\exists M) P & \neg \mathrm{I}, 2,1,8 \\ 1 & (10) & (\exists M) P & \neg \mathrm{E}, 9\end{array}$

\section{$3 \quad$ LK-Quarc $_{B}$}

We now move to the presentation of the sequent-calculus version of Quarc $_{B}$, called LK-Quarc $_{B}$. LK-Quarc ${ }_{B}$ is an adaptation of the system LK from [7]. The system presented here consists of sequents of the form $\Gamma \Rightarrow \Delta$, where $\Gamma$ and $\Delta$ are sequences of formulas, connected into derivations via derivation rules. These rules take one or more (usually two), sequents, called the upper sequent(s) and produce a single sequent, called the lower sequent. A single application of a derivation rule will be referred to as an inference.

Derivation rules are divided into five types: (i) axioms, (ii) structural, (iii) propositional, (iv) quantification and (v) special. Axioms are the initial sequents of a derivation. Structural rules concern the addition, removal or transposition of formulas in a sequent. Propositional rules concern the addition or removal of propositional (truth-functional) connectives from the lower sequent of an inference, quantification rules do he same for quantifiers, and special for reordered predicates, anaphora and negative predication. Finally, the Cut rule, although a structural rule, is listed separately, as it will be a rule we will eliminate in subsequent sections.

Every rule of LK-Quarc ${ }_{B}$, with the exception of Cut operates either on the left (marked by L before the relevant symbol), or the right (R) side of the arrow in the lower sequent. As we will see later, LK-Quarc 2 and LK-Quarc 3 will offer further exceptions to this convention.

The sequent which is not an upper sequent of an inference is called an endsequent of a derivation it belongs to. A derivation can have only one endsequent, as will be obvious from the structure of the derivation rules. We now proceed to define them.

Definition 7 (LK-Quarc ${ }_{B}$ ) The following are the rules of LK-Quarc ${ }_{B}$. In all but the Cut rule, the formula occurring in the lower sequent of a rule other than $\Gamma$ and $\Delta$ is called the principal formula of that rule. 


\subsection{Axioms}

An axiom is a sequent of the form $\left(t_{1}, \ldots, t_{n}\right) P \Longrightarrow\left(t_{1}, \ldots, t_{n}\right) P$, where $t_{1}, \ldots, t_{n}$ are singular arguments and $P$ is a $n$-ary predicate. Axioms are also called initial sequents, given that they are not a lower sequent of any inference.

\subsection{Structural}

We next define the structural rules. As stated previously, these rules govern the addition (weakening, $W$ ), removal (contraction, $C$ ), and transposition (exchange, $P$ ) of formulas in the lower sequent.

1. $\frac{\Gamma \Longrightarrow \Delta}{A, \Gamma \Longrightarrow \Delta}(\mathrm{L} W) \stackrel{\Gamma \Longrightarrow \Delta}{\Gamma \Longrightarrow \Delta, A}(\mathrm{R} W)$

2. $\frac{A, A, \Gamma \Longrightarrow \Delta}{A, \Gamma \Longrightarrow \Delta}(\mathrm{L} C) \quad \frac{\Gamma \Longrightarrow \Delta, A, A}{\Gamma \Longrightarrow \Delta, A}(\mathrm{R} C)$

3. $\frac{\Gamma^{\prime}, A, B, \Gamma \Longrightarrow \Delta}{\Gamma^{\prime}, B, A, \Gamma \Longrightarrow \Delta}(\mathrm{L} P) \quad \frac{\Gamma \Longrightarrow \Delta, A, B, \Delta^{\prime}}{\Gamma \Longrightarrow \Delta, B, A, \Delta^{\prime}}(\mathrm{R} P)$

\subsection{Propositional}

The rules in this section do not introduce anything unfamiliar to those acquainted with standard LK. Therefore, in a number of subsequent section segments concerning these rules will be omitted or presented only schematically.

1. $\frac{\Gamma \Longrightarrow \Delta, A}{\neg A, \Gamma \Longrightarrow \Delta}(\mathrm{L} \neg) \quad \frac{A, \Gamma \Longrightarrow \Delta}{\Gamma \Longrightarrow \Delta, \neg A}(\mathrm{R} \neg)$

2. $\frac{A, \Gamma \Longrightarrow \Delta}{A \wedge B, \Gamma \Longrightarrow \Delta}(\mathrm{L} \wedge)^{*} \frac{\Gamma \Longrightarrow \Delta, A \quad \Gamma \Longrightarrow \Delta, B}{\Gamma \Longrightarrow \Delta, A \wedge B}(\mathrm{R} \wedge)$

3. $\frac{A, \Gamma \Longrightarrow \Delta \quad B, \Gamma \Longrightarrow \Delta}{A \vee B, \Gamma \Longrightarrow \Delta}(\mathrm{L} \vee) \frac{\Gamma \Longrightarrow \Delta, A}{\Gamma \Longrightarrow \Delta, A \vee B}(\mathrm{R} \vee)^{*}$ 
4. $\frac{B, \Gamma \Longrightarrow \Delta \quad \Gamma \Longrightarrow \Delta, A}{A \rightarrow B, \Gamma \Longrightarrow \Delta}(\mathrm{L} \rightarrow) \quad \frac{A, \Gamma \Longrightarrow \Delta, B}{\Gamma \Longrightarrow \Delta, A \rightarrow B}(\mathrm{R} \rightarrow)$

* - the rules $\mathrm{L} \wedge$ and $\mathrm{R} \vee$ can also, respectively, produce the formula $B \wedge A$ and $B \vee A$.

\subsection{Quantification}

The primary novelty of Quarc is in its treatment of Quantified Arguments. Therefore, the rules in this section will constitute (along with the Cut rule) the primary focus of this paper.

$$
\begin{aligned}
& \text { 1. } \frac{A[a / \forall M], \Gamma \Longrightarrow \Delta \quad \Gamma \Longrightarrow \Delta, a M}{A[\forall M], \Gamma \Longrightarrow \Delta}(\mathrm{L} \forall) \quad \frac{a M, \Gamma \Longrightarrow \Delta, A[a / \forall M]}{\Gamma \Longrightarrow \Delta, A[\forall M]}(\mathrm{R} \forall)^{*} \\
& \text { 2. } \frac{a M, A[a / \exists M], \Gamma \Longrightarrow \Delta}{A[\exists M], \Gamma \Longrightarrow \Delta}(\mathrm{L} \exists)^{*} \quad \frac{\Gamma \Longrightarrow \Delta, a M \quad \Gamma \Longrightarrow \Delta, A[a / \exists M]}{\Gamma \Longrightarrow \Delta, A[\exists M]}(\mathrm{R} \exists) \\
& \text { * - the Singular Argument } a \text { does not occur anywhere in } \Gamma, \Delta, A[\forall M] \text { or } \\
& A[\exists M] .
\end{aligned}
$$

Note here that the rules of universal quantification bear a structural similarity to free logic [8]. In free logic (regardless of which version of it we consider in this context) one of its characteristic axioms is $E ! a \wedge A[a] \rightarrow \exists x A[x]$ in some Hilbert-style axiomatization. E.g. [2] formulates the rules for the introduction of the existential quantifiers for some Gentzen (sequent) system as follows (we use here a slightly simpler syntax):

$$
\begin{aligned}
& \frac{\Gamma, E ! a, A[a] \Rightarrow \Delta}{\Gamma, \exists x A[x] \Rightarrow \Delta}(\mathrm{L} \exists), a \text { does not occur below the inference line } \\
& \frac{\Gamma \Rightarrow \Delta, E ! a \quad \Gamma \Rightarrow \Delta, A[a]}{\Gamma \Rightarrow \Delta, \exists x A[x]}(\mathrm{R} \exists)
\end{aligned}
$$

Assuming that the premise of $(\mathrm{L} \exists)$ is derivable, then so is: (1) $\Gamma, E ! a \wedge A[a] \Rightarrow \Delta$; likewise if both premises of $(\mathrm{R} \exists)$ are derivable, then so is: (2) $\Gamma \Rightarrow \Delta, E ! a \wedge$ $A[a]$. On the other hand, these rules express syntactically that the existential quantifier has (in this case) existential import. This is made clearer by the fact that the following sequent is derivable in (positive and negative) free logic: $E ! a \wedge A[a] \Rightarrow \exists x A[x]$. From a proof-theoretic semantics position this might 
be seen as a disadvantage, since there is implicitly conjunction introduction involved.

However, one of the main aims of this paper is to establish the cut elimination theorem for (several variants of) Quarc, and the authors are not aware of any paper in the tradition of free logic that formulates the rules for $(\mathrm{L} \exists)$ and $(\mathrm{R} \exists)$ in a way more truthful to proof theoretic semantics; i.e. such that the formulations of both rules do not tacitly rely on conjunction introduction (on both sides) but in such a way that the crucial sequent $E ! a \wedge A[a] \Rightarrow \exists x A[x]$ is still derivable. This could in fact stimulate another paper that addresses this is issue.

\subsection{Special}

This section introduces further rules (in addition to those for quantification) specific to Quarc, those for anaphora, reorder and negative predication. Here $R$ is an $n$-ary predicate, $R^{\pi}$ is a reordered $n$-ary predicate and $P$ is either an $n$-ary predicate, or a reordered $n$-ary predicate.

1. $\frac{A\left[\ldots a_{1} \ldots a_{n} \ldots\right], \Gamma \Longrightarrow \Delta}{A\left[\ldots a_{\alpha} / a_{1} \ldots \alpha / a_{n} \ldots\right], \Gamma \Longrightarrow \Delta}(\mathrm{L} A) \quad \frac{\Gamma \Longrightarrow \Delta, A\left[\ldots a_{1} \ldots a_{n} \ldots\right]}{\Gamma \Longrightarrow \Delta, A\left[\ldots a_{\alpha} / a_{1} \ldots \alpha / a_{n} \ldots\right]}(\mathrm{R} A)$

2. $\frac{\left(t_{1}, \ldots, t_{n}\right) R, \Gamma \Longrightarrow \Delta}{\left(t_{\pi 1}, \ldots, t_{\pi n}\right) R^{\pi}, \Gamma \Longrightarrow \Delta}(\mathrm{L} R d) \quad \frac{\Gamma \Longrightarrow \Delta,\left(t_{1}, \ldots, t_{n}\right) R}{\Gamma \Longrightarrow \Delta,\left(t_{\pi 1}, \ldots, t_{\pi n}\right) R^{\pi}}(\mathrm{R} R d)$

3. $\frac{\neg\left(t_{1}, \ldots, t_{n}\right) P, \Gamma \Longrightarrow \Delta}{\left(t_{1}, \ldots, t_{n}\right) \neg P, \Gamma \Longrightarrow \Delta}(\mathrm{L} N P) \frac{\Gamma \Longrightarrow \Delta, \neg\left(t_{1}, \ldots, t_{n}\right) P}{\Gamma \Longrightarrow \Delta,\left(t_{1}, \ldots, t_{n}\right) \neg P}(\mathrm{R} N P)$

\subsection{Cut}

Finally, we have the Cut rule. The formula $A$ in the schema below is called the cut formula of the application of the rule.

1. $\frac{\Gamma \Rightarrow \Theta, A \quad A, \Pi \Rightarrow \Delta}{\Gamma, \Pi \Rightarrow \Theta, \Delta}$

\subsection{Axiom Generalization}

Before proceeding, let us demonstrate a simple and useful lemma - that the axiom rule, which has been defined only for the basic sentences, can be generalized for any formula $A$.

Lemma 1 All sequents of the form $A \Rightarrow A$ is derivable in LK-Quarc ${ }_{B}$. 
Proof. By induction on the terminal symbol of $A$.

Basic step. Every initial sequent is derivable.

Inductive step. Here we will only examine, as an illustration, the example of the universal quantifier.

1. Universal Quantifier

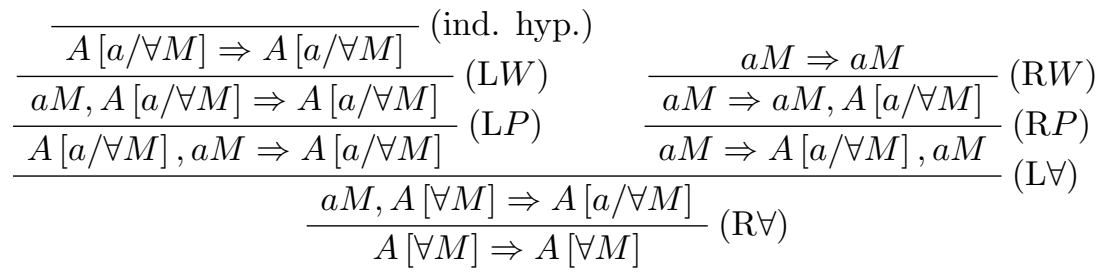

Where $a$ is some singular argument such that $A[\forall M]$ does not contain it.

\section{Deductive Equivalence}

In this section we will demonstrate the deductive equivalence of LK-Quarc ${ }_{B}$ and $\operatorname{Quarc}_{B}$. Note that we will make full use of the Cut rule (even though the Cut Elimination Theorem will later guarantee that for each derivation presented here, there is a cut-free derivation).

Before proceeding, a note on the structure of this section may perhaps be helpful. Theorem 2 is demonstrated by proving two auxiliary lemmas, Lemma 2 and Lemma 3, each corresponding to one direction of the biconditional in Theorem 2. The proof of basic step of Lemma 3 is Lemma 1 and the inductive step of Lemma 3 for the Universal Elimination requires the (trivial) Lemma 4.

Theorem 2 LK-Quarc ${ }_{B}$ and $\operatorname{Quarc}_{B}$ are deductively equivalent. Namely, every endsequent of any derivation of LK-Quarc ${ }_{B}$ is derivable in Quarc $_{B}$, and for any line (i) of any proof in $\mathrm{Quarc}_{B}$ there exists a corresponding sequent in LKQuarc $_{B}$ which can be derived from trivial lemmas and sequents corresponding to the lines of a proof (i) is derived from in $\operatorname{Quarc}_{B}$.

Obviously, what needs to be explained first is what the correspondence between the lines of a proof and sequents is. To do that, we define the standard translation:

Definition 8 (Standard Translation) Standard translation of a sequent $\Gamma \Rightarrow \Delta$ of LK-Quarc, where $\Gamma=\left\{\gamma_{1}, \ldots, \gamma_{n}\right\}$ and $\Delta=\left\{\delta_{1}, \ldots, \delta_{m}\right\}$ is the derivation in Quarc $\gamma_{1} \wedge \ldots \wedge \gamma_{n} \vdash \delta_{1} \vee \ldots \vee \delta_{m}$. Conversely, standard translation of a line of a proof in Quarc $\langle\Gamma,(i), \delta, R\rangle$ is the sequent $\Gamma \Rightarrow \delta$.

The proof of the theorem proceeds through proof of two lemmas, one going from the LK-Quarc ${ }_{B}$ to Quarc $_{B}$, and the other in the opposite direction. 


\subsection{From LK-Quarc to Quarc}

The proof in this direction goes by the following lemma:

Lemma 2 Every endsequent $\Gamma \Rightarrow \Delta$ of some derivation in LK-Quarc ${ }_{B}$ is, given standard translation, derivable in $\operatorname{Quarc}_{B}$.

Proof. By induction on applications of rules of LK-Quarc ${ }_{B}$.

Basic step. Every initial sequent is derivable in Quarc $_{B}$. Follows trivially from the Premise rule of Quarc.

Inductive step. Henceforth, we outline the important steps.

1. (L $\forall$ ) Assume that in $\operatorname{Quarc}_{B}$ (i) $A[a / \forall M] \wedge \Gamma \vdash \Delta$ and (ii) $\Gamma \vdash \Delta \vee a M$. Now assume (1) $A[\forall M] \wedge \Gamma$. We need derive $\Delta$.

$\begin{array}{rcll}1 & (1) & A[\forall M] \wedge \Gamma & \text { Premise } \\ 1 & (2) & A[\forall M] & \wedge \mathrm{E}, 1 \\ 1 & (3) & \Gamma & \wedge \mathrm{E}, 1 \\ 1 & (4) & \Delta \vee a M & \text { by (ii) } \\ 5 & (5) & \Delta & \text { Premise } \\ 6 & (6) & a M & \text { Premise } \\ 1,6 & (7) & A[a / \forall M] & \text { UE, } 2,6 \\ 1,6 & (8) & A[a / \forall M] \wedge \Gamma & \wedge \mathrm{I}, 7,3 \\ 1,6 & (9) & \Delta & \text { by (i) } \\ 1 & (10) & \Delta & \vee \mathrm{E}, 4,5,5,6,9\end{array}$

2. $(\mathrm{R} \forall)$ Assume that in $\operatorname{Quarc}_{B}$ (i) $a M \wedge \Gamma \vdash \Delta \vee A[a / \forall M]$ and (ii) $a$ does not appear anywhere in $\Gamma, \Delta$ or $A[\forall M]$. Now assume (1) $\Gamma$. We need to derive $\Delta \vee A[\forall M]$.

$\begin{array}{rcll}1 & (1) & \Gamma & \text { Premise } \\ 2 & (2) & a M & \text { Premise } \\ 1,2 & (3) & a M \wedge \Gamma & \wedge \mathrm{I}, 1,2 \\ 1,2 & (4) & \Delta \vee A[a / \forall M] & \text { by (i) } \\ & (5) & \Delta \vee \neg \Delta & \text { Prop. } \\ 6 & (6) & \Delta & \text { Premise } \\ 6 & (7) & \Delta \vee A[\forall M] & \vee \mathrm{I}, 6 \\ 8 & (8) & \neg \Delta & \text { Premise } \\ 1,2,8 & (9) & A[a / \forall M] & \text { Prop. } 4,8 \\ 1,8 & (10) & A[\forall M] & \text { UI, } 2,9, \text { given (ii) } \\ 1,8 & (11) & \Delta \vee A[\forall M] & \vee \mathrm{I}, 10 \\ 1 & (12) & \Delta \vee A[\forall M] & \vee \mathrm{E}, 5,6,7,8,11\end{array}$


3. (L $A)$ Assume (i) $A\left[\ldots a_{1} \ldots a_{n} \ldots\right] \wedge \Gamma \vdash \Delta$ and assume (1) $A\left[a_{\alpha} / a_{1} \ldots \alpha / a_{n}\right] \wedge$ $\Gamma$. We need to derive $\Delta$.

$\begin{array}{llll}1 & (1) & A\left[a_{\alpha} / a_{1} \ldots \alpha / a_{n} \ldots\right] \wedge \Gamma & \text { Premise } \\ 1 & (2) & A\left[a_{\alpha} / a_{1} \ldots \alpha / a_{n} \ldots\right] & \wedge \mathrm{E}, 1 \\ 1 & (3) & A\left[\ldots a_{1} \ldots a_{n} \ldots\right] & \mathrm{AE}, 2 \\ 1 & (4) & \Gamma & \wedge \mathrm{E}, 1 \\ 1 & (5) & A\left[a_{1} \ldots a_{n} \ldots\right] \wedge \Gamma & \wedge \mathrm{I}, 3,4 \\ 1 & (6) & \Delta & \text { by }(\mathrm{i})\end{array}$

Obviously, this is straightforward.

4. Similarly for other Special rules.

This concludes the proof of Lemma 2. We now turn to the proof of the other Lemma.

\subsection{From Quarc to LK-Quarc}

In this direction the proof relies on the following lemma:

Lemma 3 For any line (i) of any proof in $\operatorname{Quarc}_{B}$ there exists a corresponding sequent in $\mathrm{LK}$-Quarc $_{B}$ which can be derived from trivial lemmas and sequents corresponding to the lines of a proof (i) is derived from in $\operatorname{Quarc}_{B}$.

Before proceeding with the proof, perhaps a slight clarification of this lemma is in order. Keep in mind that every step of a proof in Quarc is derived from previous step or steps (or none for Premise and Identity Introduction) via the application of a certain rule. What this lemma does is construct a segment of a derivation in LK-Quarc ${ }_{B}$ (not a full derivation because it does not necessarily have an initial sequent in all of its topmost places) that begins with the (standard translation of) steps the application of the rule of Quarc $_{B}$ relies on, and ends with the (standard translation of) step that the rule produces.

Since any proof in Quarc consist of a finite number of steps each produced by a rule, by "stacking" the segments of the derivation one after the other (one segment for each step, according to the rule used in that step), we produce a derivation for which the endsequent is the standard translation of the conclusion of the proof in Quarc $_{B}$. We now proceed with the proof of the lemma.

Proof. By induction on the applications of the rules of derivation of $\mathrm{Quarc}_{B}$.

Basic step. Since we are dealing with $\operatorname{Quarc}_{B}$, which does not include the identity rules, a proof can only begin with an application of a Premise rule. For any application of the Premise rule, the corresponding sequent is $A \Rightarrow A$. That such a sequent exists is shown by Lemma 1 .

Inductive step. 
1. $(\neg \mathrm{I})$ The rule for the Negation Introduction has the following form:

$$
\begin{array}{rccl}
\mathrm{k} & (\mathrm{k}) & A & \text { Premise } \\
\mathrm{L}_{1} & (\mathrm{~m}) & B & \\
\mathrm{~L}_{2} & (\mathrm{n}) & \neg B & \\
\mathrm{~L}_{1} *, \mathrm{~L}_{2}{ }^{*} & (\mathrm{i}) & \neg A & \neg \mathrm{I}, \mathrm{k}, \mathrm{m}, \mathrm{n}
\end{array}
$$

Here $\mathrm{L}_{n}{ }^{*}$ stands for the sequence of formulas $\mathrm{L}_{n}$ with all the occurences of $k$ omitted.

The corresponding segment of a derivation in LK-Quarc ${ }_{B}$ is as follows (part separated out for legibility):

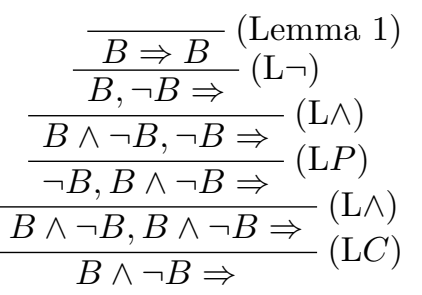

We now use this part in the top right and provide the rest of the segment:

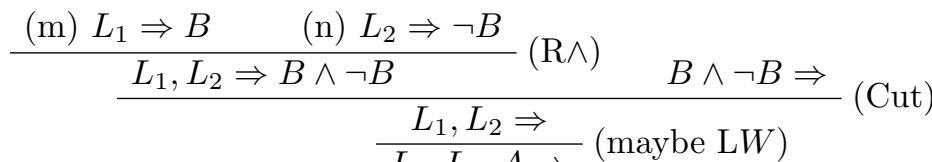

$$
\begin{aligned}
& \text { (k) } A \Rightarrow A \quad \frac{L_{1}, L_{2}, A \Rightarrow}{L_{1} *, L_{2} *, A \Rightarrow} \text { (maybe some } \mathrm{L} C \text { ) } \\
& \frac{L_{1} *, L_{2} *, A \Rightarrow}{\text { (i) } L_{1} *, L_{2} * \Rightarrow \neg A}(\mathrm{R} \neg)
\end{aligned}
$$

Obviously, here we could do without the sequent corresponding to the step (k) and the application of Cut it is a part of, but we use all the steps that are listed in the justification of the application of the rule in Quarc, regardless of whether they are premises or not.

These derivations are schematic. For instance, the inference between the sequents $L_{1}, L_{2} \Rightarrow$ and $L_{1}, L_{2}, A \Rightarrow$ may require a use of the left weakening rule ( $\mathrm{L} W$ ) in case neither $L_{1}$ nor $L_{2}$ contain $A$. If they do, this step can be omitted. Similarly, if either $L_{1}$ or $L_{2}$ contain $A$, one or more applications of the left contraction ( $\mathrm{L} C$ ) rule may be required to obtain the sequent $L_{1} *, L_{2} *, A \Rightarrow$. Again, in case neither $L_{1}$ nor $L_{2}$ contain $A$ these steps can be omitted.

2. Similarly for other propositional rules.

3. (UE) The rule for the Universal Elimination has the following form: 

$\mathrm{L}_{1} \quad(\mathrm{k}) \quad A[\forall M]$
$\mathrm{L}_{2} \quad(\mathrm{~m}) \quad a M$
$\mathrm{L}_{1}, \mathrm{~L}_{2} \quad$ (i) $\quad A[a / \forall M] \quad \mathrm{UE}, \mathrm{k}, \mathrm{m}$

Before proceeding with the corresponding segment of a derivation, we need to prove the following (easy) lemma:

Lemma 4 The sequent $A[\forall M], a M \Rightarrow A[a / \forall M]$ is derivable in LK$\operatorname{Quarc}_{B}$.

Proof.

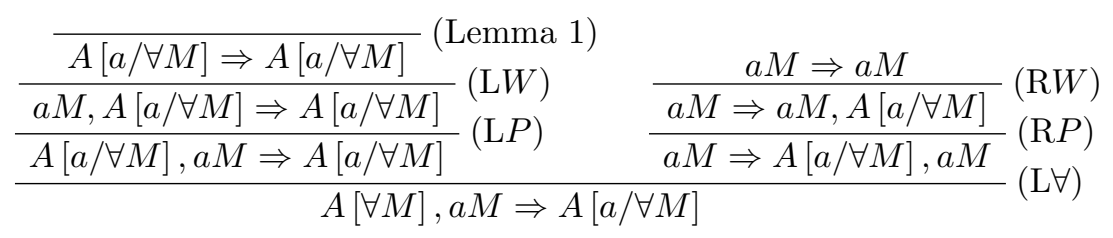

The corresponding segment of a derivation in LK-Quarc ${ }_{B}$ for the rule UE is as follows:

$$
\frac{(\mathrm{k}) L_{1} \Rightarrow A[\forall M]}{\frac{(\mathrm{m}) L_{2} \Rightarrow a M \quad \overline{A[\forall M], a M \Rightarrow A[a / \forall M]}}{L_{2}, A[\forall M] \Rightarrow A[a / \forall M]} \text { (Lemma 4) }} \text { (Cut) }
$$

4. (UI) The rule for the Universal Introduction has the following form:

$$
\begin{array}{rlll}
\mathrm{k} & (\mathrm{k}) & a M & \text { Premise } \\
\mathrm{L}_{1} & (\mathrm{~m}) & A[a / \forall M] & \\
\mathrm{L}_{1} * & (\mathrm{i}) & A[\forall M] & \mathrm{UI}, \mathrm{k}, \mathrm{m}
\end{array}
$$

Here $\mathrm{L}_{1}$ * stands for the sequence of formulas $\mathrm{L}_{1}$ with all the occurrences of $k$ omitted. By rule, $\mathrm{L}_{1}$ contains no occurrences of the SA $a$ apart from that in $k$, and therefore $\mathrm{L}_{1} *$ contains no occurrences of $a$.

The corresponding segment of a derivation in LK-Quarc ${ }_{B}$ for the rule UI is as follows:

$$
\frac{\frac{(\mathrm{m}) L_{1} \Rightarrow A[a / \forall M]}{a M, L_{1} \Rightarrow A[a / \forall M]}}{(\mathrm{k}) a M \Rightarrow a M \quad \frac{\mathrm{L} W}{a M, L_{1} * \Rightarrow A[a / \forall M]}}(\text { maybe LC) }
$$


Since $\mathrm{L}_{1}{ }^{*}$ contains no occurrences of $a$, this is an appropriate use of the rule $\mathrm{R} \forall$.

5. (PI) The rule for the Particular Introduction has the following form:

$$
\begin{array}{rcll}
L_{1} & (\mathrm{k}) & A[a / \exists M] & \\
L_{2} & (\mathrm{~m}) & (a) M & \\
L_{1}, L_{2} & \text { (i) } & A[\exists M] & \text { PI, i, j }
\end{array}
$$

The corresponding segment of a derivation in LK-Quarc ${ }_{B}$ for the rule PI is as follows:

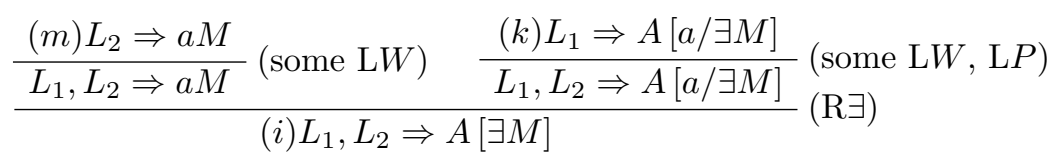

6. (PE) The rule for the Particular Elimination has the following form:

$$
\begin{array}{rcll}
L_{1} & (\mathrm{k}) & A[\exists M] & \\
j & (\mathrm{l}) & (a) M & \text { Premise } \\
k & (\mathrm{~m}) & A[a / \exists M] & \text { Premise } \\
L_{2} & (\mathrm{n}) & B & \\
L_{1}, L_{2}-\{j, k\} & (\mathrm{i}) & B & \text { PE, } \mathrm{k}, \mathrm{l}, \mathrm{m}, \mathrm{n}
\end{array}
$$

The singular argument $a$ occurs nowhere in $L_{1}, A[\exists M]$ or $B$, and nowhere in $L_{2}$ except j or $\mathrm{k}$.

The corresponding segment of a derivation in LK-Quarc ${ }_{B}$ for the rule PE is as follows (broken into two parts for legibility):

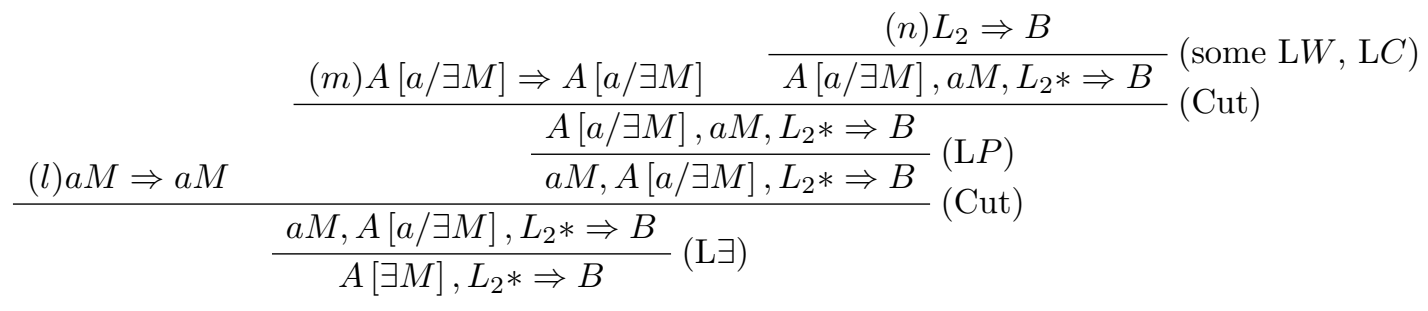

where $L_{2} *$ stands for the sequence of formulas $L_{2}$ with all instances of $a M$ and $A[a / \exists M]$ removed. Since $L_{2} *$ and $B$ contain no instances of SA $a$, this is an appropriate use of the rule $\mathrm{L} \exists$. Now, having obtained the sequent $A[\exists M], L_{2} * \Rightarrow B$, we combine it with the step (k) and obtain the desired sequent:

$$
\frac{(k) L_{1} \Rightarrow A[\exists M] \quad A[\exists M], L_{2} * \Rightarrow B}{(i) L_{1}, L_{2} * \Rightarrow B}(\mathrm{Cut})
$$


7. The derivations of sequents corresponding to the special symbols of Quarc are trivial and will be omitted here

This concludes the proof of Lemma 3 and thus of Theorem 2 .

\section{Cut Elimination Theorem}

We finally arrive at the central section of this paper, the demonstration of the Cut elimination theorem for LK-Quarc ${ }_{B}$. This, in turn, will allow us to arrive at the subformula property for our system and motivate some further considerations in the following sections.

\subsection{Preliminaries}

The proof presented in this section is an adaptation of Gentzen's original cut elimination proof from [7]. It is a double induction on the grade and rank of the cut formula.

\subsubsection{Cut and Mix}

Since LK-Quarc ${ }_{B}$ contains the contraction rules, there might be multiple instances of the cut formula occurring. In order to be able to cut on all of those, let us also define the mix rule:

Definition 9 (Mix rule)

$$
\frac{\Gamma \Rightarrow \Theta \quad \Pi \Rightarrow \Delta}{\Gamma, \Pi^{*} \Rightarrow \Theta^{*}, \Delta}
$$

Where some formula $M$, called the mix formula occurs at least once in $\Pi$ and $\Theta$, and $\Pi^{*}$ and $\Theta^{*}$ are obtained by removing all instances of $M$ from $\Pi$ and $\Theta$, respectively.

Definition 10 ( $L K-$ Quarc $\left.^{\dagger}\right) L K-$ Quarc $^{\dagger}$ is a sequent calculus obtained from LK-Quarc by replacing the cut rule by the mix rule.

Lemma 5 For any sequent $S, S$ is provable in $L K-$ Quarc $^{\dagger}$ just in case it is provable in LK-Quarc.

Proof. By showing Cut is derivable in $L K-$ Quarc $^{\dagger}$

$$
\frac{\Gamma \Rightarrow \Theta, A \quad A, \Pi \Rightarrow \Delta}{\frac{\Gamma, \Pi^{*} \Rightarrow \Theta^{*}, \Delta}{\Gamma, \Pi \Rightarrow \Theta, \Delta}(\text { some } \mathrm{LW}, \mathrm{RW})}
$$


and conversely that Mix is derivable in LK-Quarc.

$$
\frac{\frac{\Gamma \Rightarrow \Theta}{\Gamma \Rightarrow \Theta^{*}, A}\left(\text { some RP, RC) } \frac{\Pi \Rightarrow \Delta}{A, \Pi^{*} \Rightarrow \Delta} \text { (some L } P, \mathrm{~L} C\right. \text { ) }}{\Gamma, \Pi^{*} \Rightarrow \Theta^{*}, \Delta}
$$

Since LK-Quarc ${ }_{B}$ contains all the rules used, this lemma will hold for it. We will call LK-Quarc ${ }_{B}^{\dagger}$ the sequent calculus obtained by substituting the mix rule for the cut rule in LK-Quarc $B$.

\subsubsection{Grade and Rank}

Definition 11 (Grade, $\gamma$ ) Let $A, B$ and $C$ be formulas, $R$ an $n$-ary predicate, $P$ an $n$-ary predicate or a reordered $n$-ary predicate, $t_{1}, \ldots, t_{n}$ SA's and $\pi 1, \ldots, \pi n$ some permutation of $1, \ldots, n$ except identity permutation. Then, the grade $\gamma(A)$ of the formula $A$ is:

1. $\gamma(A)=0$ if $A$ is basic.

2. $\gamma(A)=1$ if $A$ is $\left(t_{\pi 1}, \ldots, t_{\pi n}\right) R^{\pi}$.

3. $\gamma(A)=\gamma\left(\left(t_{1}, \ldots, t_{n}\right) P\right)+1$ if $A$ is $\left(t_{1}, \ldots, t_{n}\right) \neg P$.

4. $\gamma(A)=\gamma(B)+1$ if $A$ is $\neg B$.

5. $\gamma(A)=\gamma(B)+\gamma(C)+1$ if $A$ is $B \wedge C, B \vee C$ or $B \rightarrow C$.

6. $\gamma(A)=\gamma(B[t / \forall P])+1$ if $A$ is $B[\forall P]$.

7. $\gamma(A)=\gamma(B[t / \exists P])+1$ if $A$ is $B[\exists P]$.

8. $\gamma(A)=\gamma\left(B\left[\ldots, t_{1}, \ldots, t_{n}, \ldots\right]\right)+1$ if $A$ is $B\left[\ldots, t_{\alpha} / t_{1}, \ldots, \alpha / t_{n}\right]$.

The order of application of the rule for anaphora can sometimes be transposed with the application of the rules for sentential operators, quantifiers, or another anaphora. It can be shown by induction that all of those transpositions assign the same grade to a formula. For a similar proof, see [5].

Definition 12 (Rank, $\rho$ ) Rank of a derivation is the sum of the left and right rank of a mix formula. Left rank (right rank) is the maximal number of sequents in a branch, starting from the upper left (right) sequent of the mix rule, such that each sequent of the branch contains the mix formula in the succedent (antecedent). 


\subsubsection{Re-designating the Proper Singular Arguments}

Before proceeding to the cut elimination theorem, we shall prove an auxiliary lemma. Again, this is due to [7].

Definition 13 Call the Singular Argument $a$ occurring in the Definition 6 of the rules $\mathrm{R} \forall$ and $\mathrm{L} \exists$ the proper singular argument of the respective rules. To re-designate the proper singular arguments, we alter a derivation according to the following procedure. First, for every occurrence of a rule $\mathrm{R} \forall$ or $\mathrm{L} \exists$ above which no other occurrence of these rules is present (to have a unique procedure we can start with the leftmost and move right), we replace their proper singular argument in all the sequents above the lower sequent of the occurrence of the rule with a singular argument that has so far not occurred anywhere in the derivation. Second, we apply the same procedure to all the occurrences of the rules $\mathrm{R} \forall$ or $\mathrm{L} \exists$ which are such that the procedure has already been applied to any other occurrence of said rules in all the sequents above their lower sequents.

We need to prove the following auxiliary lemma:

Lemma 6 If In is an initial sequent or a correct inference which contains a singular argument $a$, which is not the proper singular argument of In, and if the singular argument $b$ is likewise not the proper singular argument of In, then In', obtained from In by uniformly substituting $b$ for $a$ is an initial sequent or a correct inference.

Proof. By induction on the rules of LK-Quarc ${ }_{B}$.

Next we prove the following lemma:

Lemma 7 If we re-designate the proper singular arguments of a correct derivation, it will yield a correct derivation, namely of the same grade and rank, of the same endsequent.

That the two derivations end in the same endsequent is obvious from the definition of the re-designation procedure. We now need to show this is a correct derivation of a said sequent.

Proof. By induction on the steps of the re-designation procedure. For every occurrence of a rule $\mathrm{R} \forall$ or $\mathrm{L} \exists$, every sequent above its lower sequent is derived correctly, by Lemma 6 and inductive hypothesis. Moreover, replacing the proper singular argument of a correct application of $\mathrm{R} \forall$ or $\mathrm{L} \exists$ with a singular argument that occurs nowhere above its lower sequent will likewise produce a correct instance of $\mathrm{R} \forall$ or $\mathrm{L} \exists$. 


\subsection{Cut Elimination}

We want to show the following:

Theorem 3 (Cut Elimination) For any sequent $S$, if $S$ is provable in LKQuarc $_{B}$, then it is provable in LK-Quarc $B$ without using the cut rule.

Given Lemma 5, it will suffice to show:

Lemma 8 For any sequent $S$, if $S$ is provable in LK-Quarc ${ }_{B}^{\dagger}$, then it is provable in LK-Quarc ${ }_{B}^{\dagger}$ without using the mix rule.

Proof. By induction on grade and rank.

\subsection{1 $\rho=2$}

Obviously, the lowest rank of an application of a mix rule is 2. So, suppose $\rho(M)=2$. We will omit all the familiar cases and focus on the symbols of Quarc.

\section{Special}

We start with the special symbols of $\mathrm{LK}-\mathrm{Quarc}_{B}$ as those have the lowest grade.

1. Reorder:

$$
\frac{\frac{\Gamma \Longrightarrow \Theta,\left(t_{1}, \ldots, t_{n}\right) R}{\Gamma \Longrightarrow \Theta,\left(t_{\pi 1}, \ldots, t_{\pi n}\right) R^{\pi}} \quad \frac{\left(t_{1}, \ldots, t_{n}\right) R, \Pi \Longrightarrow \Delta}{\left(t_{\pi 1}, \ldots, t_{\pi n}\right) R^{\pi}, \Pi \Longrightarrow \Delta}}{\Gamma, \Pi \Rightarrow \Theta, \Delta} \text { (Mix) }
$$

This can be transformed into:

$$
\frac{\Gamma \Longrightarrow \Theta,\left(t_{1}, \ldots, t_{n}\right) R \quad\left(t_{1}, \ldots, t_{n}\right) R, \Pi \Longrightarrow \Delta}{\frac{\Gamma, \Pi^{*} \Rightarrow \Theta^{*}, \Delta}{\Gamma, \Pi \Rightarrow \Theta, \Delta}(\text { some } \mathrm{R} W, \mathrm{R} P, \mathrm{~L} W, \mathrm{~L} P)}
$$

Since the mix formula is of a lower grade, by inductive hypothesis, it can be eliminated. 
2. Anaphora:

$$
\frac{\frac{\Gamma \Longrightarrow \Theta, A\left[\ldots a_{1} \ldots a_{n} \ldots\right]}{\Gamma \Longrightarrow \Theta, A\left[\ldots a_{\alpha} / a_{1} \ldots \alpha / a_{n} \ldots\right]} \quad \frac{A\left[\ldots a_{1} \ldots a_{n} \ldots\right], \Pi \Longrightarrow \Delta}{\Gamma, \Pi\left[\ldots a_{\alpha} / a_{1} \ldots \alpha / a_{n} \ldots\right], \Pi \Longrightarrow \Delta}}{\Gamma, \Pi \Theta, \Delta}
$$

This can be transformed into:

$$
\frac{\Gamma \Longrightarrow \Theta, A\left[\ldots a_{1} \ldots a_{n} \ldots\right] \quad A\left[\ldots a_{1} \ldots a_{n} \ldots\right], \Pi \Longrightarrow \Delta}{\frac{\Gamma, \Pi^{*} \Rightarrow \Theta^{*}, \Delta}{\Gamma, \Pi \Rightarrow \Theta, \Delta}(\text { some } \mathrm{R} W, \mathrm{R} P, \mathrm{~L} W, \mathrm{~L} P)}
$$

Again, since the mix formula is of a lower grade, by inductive hypothesis, it can be eliminated.

3. Negative Predication:

$$
\frac{\frac{\Gamma \Longrightarrow \Theta, \neg\left(t_{1}, \ldots, t_{n}\right) P}{\Gamma \Longrightarrow \Theta,\left(t_{1}, \ldots, t_{n}\right) \neg P} \quad \frac{\neg\left(t_{1}, \ldots, t_{n}\right) P, \Pi \Longrightarrow \Delta}{\left(t_{1}, \ldots, t_{n}\right) \neg P, \Pi \Longrightarrow \Delta}}{\Gamma, \Pi \Rightarrow \Theta, \Delta} \text { (Mix) }
$$

This can be transformed into:

$$
\frac{\Gamma \Longrightarrow \Theta, \neg\left(t_{1}, \ldots, t_{n}\right) P \quad \neg\left(t_{1}, \ldots, t_{n}\right) P, \Pi \Longrightarrow \Delta}{\frac{\Gamma, \Pi^{*} \Rightarrow \Theta^{*}, \Delta}{\Gamma, \Pi \Rightarrow \Theta, \Delta}(\text { some } \mathrm{R} W, \mathrm{R} P, \mathrm{~L} W, \mathrm{~L} P)}
$$

This mix formula can be eliminated according to the procedure for negation below.

\section{Propositional}

Cut elimination theorem for the propositional symbols is a familiar result and will be omitted here, apart from negation, which is required to finalize the cut elimination for negative predication above:

$$
\frac{\frac{A, \Gamma \Rightarrow \Theta}{\Gamma \Rightarrow \Theta, \neg A} \quad \frac{\Pi \Rightarrow \Delta, A}{\neg A, \Pi \Rightarrow \Delta}}{\Gamma, \Pi \Rightarrow \Theta, \Delta} \text { (Mix) }
$$


This can be transformed into:

$$
\frac{\Pi \Rightarrow \Delta, A \quad A, \Gamma \Rightarrow \Theta}{\frac{\Pi, \Gamma^{*} \Rightarrow \Delta^{*}, \Theta}{\Pi, \Gamma \Rightarrow \Delta, \Theta}(\text { some } \mathrm{L} W, \mathrm{~L} P, \mathrm{R} W, \mathrm{R} P)}
$$

Since the mix formula $A$ is of lesser grade than $\neg A$, by inductive hypothesis, it can be eliminated.

\section{Quantification - Universal}

Let the terminal symbol of the mix formula be a universal quantifier:

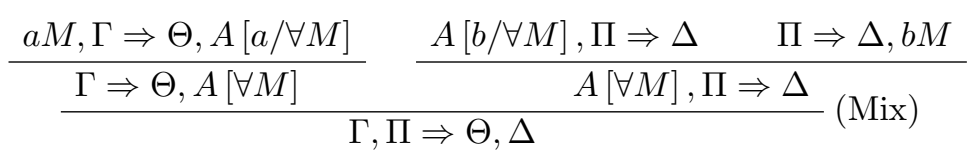

This can be transformed into:

$$
\begin{aligned}
& \frac{\Pi \Rightarrow \Delta, b M \quad b M, \Gamma \Rightarrow \Theta, A[b / \forall M]}{\Pi, \Gamma^{*} \Rightarrow \Delta^{*}, \Theta, A[b / \forall M]}(\mathrm{Mix})
\end{aligned}
$$

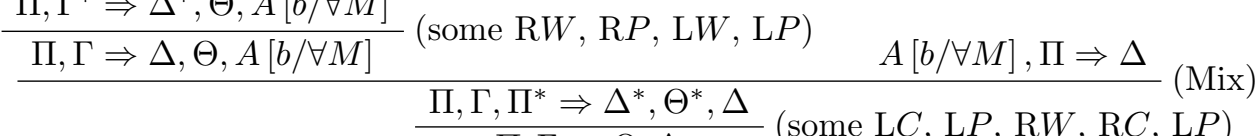

$$
\begin{aligned}
& \Pi, \Gamma \Rightarrow \Theta, \Delta \quad \text { (some } \mathrm{L} C, \mathrm{~L} P, \mathrm{R} W, \mathrm{R} C, \mathrm{~L} P \text { ) }
\end{aligned}
$$

The change from the the sequent $a M, \Gamma \Rightarrow \Theta, A[a / \forall M]$ to the sequent $b M, \Gamma \Rightarrow \Theta, A[b / \forall M]$ in the transformation above is justified by Lemma 7 .

\subsection{2 $\rho>2$}

Again, the majority of cases here are familiar results, and we focus on LK$\operatorname{Quarc}_{B}$. The only part that is not a familiar result here is $\mathrm{L} \forall$ and $\mathrm{R} \exists$, which fall under the case of two-sequent rules. In the former case, the derivation runs as follows:

1. $(\mathrm{L} \forall)$

$$
\frac{\Gamma \Rightarrow \Theta \quad \frac{A[a / \forall M], \Pi \Rightarrow \Delta \quad \Pi \Rightarrow \Delta, a M}{A[\forall M], \Pi \Rightarrow \Delta}(\mathrm{Mix})}{\Gamma, \Pi^{*}, A[\forall M] \Rightarrow \Theta^{*}, \Delta}
$$

This is transformed into: 


$$
\frac{\Gamma \Rightarrow \Theta \quad A[a / \forall M], \Pi \Rightarrow \Delta}{\frac{\Gamma, \Pi^{*}, A[a / \forall M] \Rightarrow \Theta^{*}, \Delta}{\Gamma}(\operatorname{Mix}) \quad \frac{\Gamma \Rightarrow \Theta \quad \Pi \Rightarrow \Delta, a M}{\Gamma, \Pi^{*} \Rightarrow \Theta^{*}, \Delta, a M}} \text { (Mix) }
$$

As each instance of a mix rule has rank lowered by 1 , so by the inductive hypothesis both can be eliminated. We now proceed to examine the case of $\mathrm{R} \exists$.

2. $(\mathrm{R} \exists)$

$$
\frac{\Gamma \Rightarrow \Theta \quad \frac{\Pi \Rightarrow \Delta, a M \quad \Pi \Rightarrow \Delta, A[a / \exists M]}{\Pi \Rightarrow \Delta, A[a / \exists M]}(\text { Mix })}{\Gamma, \Pi^{*} \Rightarrow \Theta^{*}, \Delta, A[\exists M]}
$$

This is transformed into:

$$
\frac{\frac{\Gamma \Rightarrow \Theta \quad \Pi \Rightarrow \Delta, a M}{\Gamma, \Pi^{*} \Rightarrow \Theta^{*}, \Delta, a M} \text { (Mix) } \quad \frac{\Gamma \Rightarrow \Theta \quad \Pi \Rightarrow \Delta, A[a / \exists M]}{\Gamma, \Pi^{*} \Rightarrow \Theta^{*}, \Delta, A[a / \exists M]}}{\Gamma, \Pi^{*} \Rightarrow \Theta^{*}, \Delta, A[\exists M]} \text { (Mix) }
$$

Again, the rank of each instance of a mix rule has been lowered by 1 , and by the inductive hypothesis both can be eliminated.

Similarly if the left rank is greater than 1 or if both are greater. This concludes the proof of the Cut elimination theorem.

\subsection{Subformula Property}

In this section we demonstrate that LK-Quarc ${ }_{B}$ possesses the subformula property. Important to note here is that no formula containing a reordered predicate is basic (it is an operation on predicates of a basic formula - compare with the definition of the grade of a formula).

Definition 14 (Subformula)

1. Every formula is a subformula of itself.

2. The formula $\left(t_{1}, \ldots, t_{n}\right) R$ is a subformula of $\left(t_{\pi 1}, \ldots, t_{\pi n}\right) R^{\pi}$.

3. The formula $\neg\left(t_{1}, \ldots, t_{n}\right) P$ is a subformula of $\left(t_{1}, \ldots, t_{n}\right) \neg P{ }^{2}$

\footnotetext{
${ }^{2}$ It might not be readily obvious why the formula on the left is the subformula of the one on the right in parts 2 and 3. To clarify this, let us first note that Quarc uses predicates in ways one does not encounter in the Predicate Calculus - primarily by using predicates in Quantified arguments, but also by employing Reorder and Negative Predication. First order logic does not contain different types of predicates nor modes of predication, so naturally these will stand apart.

In this case, the formulas on the right result from applications of operations to a predicate it is either predicated negatively, or substituted for a reordered one. The underlying intuition here is of a syntactic operation being applied to its parts to produce the resulting formula.
} 
4. Every formula $A$ and $B$ mentioned in the antecedent of the rules for generation of a formula in Definition 2 is a subformula of that formula. Moreover, any formula $t M$ is likewise the subformula of the formula $A[q M]$.

5. If a formula $A$ is a subformula of any subformula of $B$, then it is a subformula of $B$.

Theorem 4 (Subformula property) Any formula appearing in any cut-free proof of LK-Quarc ${ }_{B}$, is a subformula of some formula in its endsequent.

Proof. We only need to show that the subformula property holds for all rules of LK-Quarc ${ }_{B}$, except cut, which can be eliminated. Since this is a familiar result for the propositional and structural rules, what remains to be shown is that it holds for the quantification and special rules of LK-Quarc ${ }_{B}$.

Observing the rules for the universal quantifier:

$$
\frac{A[t / \forall M], \Gamma \Longrightarrow \Delta \quad \Gamma \Longrightarrow \Delta, t M}{A[\forall M], \Gamma \Longrightarrow \Delta}(\mathrm{L} \forall) \quad \frac{t M, \Gamma \Longrightarrow \Delta, A[t / \forall M]}{\Gamma \Longrightarrow \Delta, A[\forall M]}(\mathrm{R} \forall)^{*}
$$

We can see that any formula of $\Gamma$ and $\Delta$ will be a subformula of some formula of $\Gamma$ and $\Delta$ in the lower sequent, namely itself. Moreover, $t M$ and $A[t / \forall M]$ are both subformulas of $A[\forall M]$. Therefore, the subformula property holds for this derivation. The proof for the particular quantifier proceeds in the same manner, and is straightforward for the special symbols of Quarc.

\subsubsection{Consistency}

Given the definition of consistency,

Definition 15 (Consistency) A sequent calculus is consistent just in case the sequent $\cdots \Rightarrow \cdots$ is not derivable.

An important corollary from Theorem 4 immediately follows:

Corollary 1 LK-Quarc ${ }_{B}$ is consistent.

To see this, one need only observe that no formula is a subformula of an empty sequent.

\section{$6 \quad$ Identity}

In this section we expand LK-Quarc ${ }_{B}$ into LK-Quarc 2 by adding the two identity rules. Identity, $=$, is a binary predicate, albeit with an infix notation, and obeys all the stipulations from Definitions 1 and 2. Most notably, any formula containing only it and singular arguments is basic. 
Moreover, Definition 4 is extended by the stipulations that

8. Every formula of the form $a=a$ is true.

9. If $a=b$ is true and the formula $A\left[b_{1}, \ldots, b_{n}\right]$ is a basic formula containing the instances $b_{1}, \ldots, b_{n}$ of an SA $b$, then $A\left[a / b_{1}, \ldots, a / b_{n}\right]$ is true if $A\left[b_{1}, \ldots, b_{n}\right]$ is true.

\subsection{Identity Rules}

We now give the rules for identity. They are defined only for basic formulas (containing only singular arguments), but it can be shown inductively they generalize to any formula, following the format in which they are introduced in [5], which itself meshes seamlessly with the treatment of identity in [13].

Identity Introduction, $=\mathrm{I}$

(k) $a=a \quad=\mathrm{I}$

Identity Elimination, $=\mathrm{E}$

Let $A[b]$ be a basic formula containing occurrences $b_{1}, \ldots, b_{n}$ of a singular argument $b$ ( $A$ might also contain further occurrences of $b$ ).

$$
\begin{array}{rll}
L_{1} & (\mathrm{k}) & A[b] \\
L_{2} & (\mathrm{~m}) & a=b \\
L_{1}, L_{2} & (\mathrm{n}) & A\left[a / b_{1}, \ldots, a / b_{n}\right]
\end{array}
$$

To expand LK-Quarc ${ }_{B}$ into LK-Quarc 2 we add the following rules:

$$
\frac{a=a, \Gamma \Rightarrow \Delta}{\Gamma \Rightarrow \Delta}\left(={ }_{1}\right) \quad \frac{A[b], a=b, A[a / b], \Gamma \Rightarrow \Delta}{a=b, A[a / b], \Gamma \Rightarrow \Delta}(=2)
$$

where $A$ is a basic formula and $A[a / b]$ is a formula produced by substituting any number of occurrences of the singular argument $b$ by $a$. These rules are adjusted from those presented in [13], and chosen for technical reasons (allowing straightforward cut-elimination procedure).

Before proceeding, let us prove a simple and useful lemma.

Lemma $9 \frac{a=b, \Gamma \Rightarrow \Delta}{b=a, \Gamma \Rightarrow \Delta}$

Proof.

$$
\begin{gathered}
\frac{a=b, \Gamma \Rightarrow \Delta}{a=b, b=a, b=b, \Gamma \Rightarrow \Delta} \\
\frac{b=a, b=b, \Gamma \Rightarrow \Delta}{\frac{b=b, b=a, \Gamma \Rightarrow \Delta}{b=a, \Gamma \Rightarrow \Delta}(\mathrm{L} P)}\left({ }_{2}\right) \\
(=1)
\end{gathered}
$$




\subsubsection{Generalization of Identity Rules}

As mentioned, the rules in LK-Quarc 2 , just like in Quarc, are defined only for the basic formulas. We will now show that these rules generalize to any formula.

Theorem 5 (Identity Generalization)

For any formula $S$ of Quarc,

$$
\frac{S[b], a=b, S[a / b], \Gamma \Rightarrow \Delta}{a=b, S[a / b], \Gamma \Rightarrow \Delta}
$$

Proof. By induction on the terminal symbol of $S$. Basic step is trivial, so we proceed to the inductive step, and only examine the interesting step of the universal quantifier. In the following section $A$ need not stand for a basic formula.

Let $S$ be $A[\forall M]$. Assume (i) that the sequent $A[\forall M][b], a=b, A[\forall M][a / b], \Gamma \Rightarrow$ $\Delta$ is derivable. From (i) it follows that the sequents (ii) $A[c / \forall M][b], a=$ $b, A[\forall M][a / b], \Gamma^{\prime} \Rightarrow \Delta^{\prime}$ and (iii) $a=b, A[\forall M][a / b], \Gamma^{\prime} \Rightarrow \Delta^{\prime}, c M$ are derivable. We need to show the sequent $a=b, A[\forall M][a / b], \Gamma^{\prime} \Rightarrow \Delta^{\prime}$ is derivable. The derivation proceeds as follows, broken into parts for legibility:

$$
\frac{A[c / \forall M][b] \Rightarrow A[c / \forall M][b]}{\frac{A[c / \forall M][b], a=b, A[c / \forall M][a / b] \Rightarrow A[c / \forall M][b]}{a=b, A[c / \forall M][a / b] \Rightarrow A[c / \forall M][b]}} \text { (some LW, LP } \text { (Ind. Hyp.) }
$$

We now proceed by using this sequent as the upper left sequent of the following cut, also utilizing (ii):

$$
\frac{a=b, A[c / \forall M][a / b] \Rightarrow A[c / \forall M][b] \quad \text { (ii) } A[c / \forall M][b], a=b, A[\forall M][a / b], \Gamma^{\prime} \Rightarrow \Delta^{\prime}}{\frac{a=b, A[c / \forall M][a / b], a=b, A[\forall M][a / b], \Gamma^{\prime} \Rightarrow \Delta^{\prime}}{A[c / \forall M][a / b], a=b, A[\forall M][a / b], \Gamma^{\prime} \Rightarrow \Delta^{\prime}}(\mathrm{L} P, \mathrm{~L} C)}
$$

Next, we use this sequent as the upper left sequent of L $\forall$, also utilizing (iii):

$$
\frac{A[c / \forall M][a / b], a=b, A[\forall M][a / b], \Gamma^{\prime} \Rightarrow \Delta^{\prime} \quad(\text { iii }) a=b, A[\forall M][a / b], \Gamma^{\prime} \Rightarrow \Delta^{\prime}, c M}{\frac{A[\forall M][a / b], a=b, A[\forall M][a / b], \Gamma^{\prime} \Rightarrow \Delta^{\prime}}{a=b, A[\forall M][a / b], \Gamma \Rightarrow \Delta}(\mathrm{L} P, \mathrm{~L} C)}
$$

This concludes the proof of the Theorem 5 .

\subsection{Deductive Equivalence}

The proof of deductive equivalence proceeds with the expansion of the proof of Theorem 2 with the appropriate steps for the identity rules. 


\subsubsection{LK-Quarc to Quarc}

1. $(=1)$ Assume that in $\mathrm{Quarc}_{2}$ (i) $a=a \wedge \Gamma \vdash \Delta$. Now assume (1) $\Gamma$. We need to derive $\Delta$.

$\begin{array}{llll}1 & (1) & \Gamma & \text { Premise } \\ & (2) & a=a & =\mathrm{I} \\ 1 & (3) & a=a \wedge \Gamma & \wedge \mathrm{I}, 1,2 \\ 1 & (4) & \Delta & \text { by (i) }\end{array}$

2. $(=2)$ Assume that in $\operatorname{Quarc}_{2}$ (i) $A[b] \wedge a=b \wedge A[a / b] \wedge \Gamma \vdash \Delta$. Now assume (1) $a=b \wedge A[a / b] \wedge \Gamma$. We need to derive $\Delta$.

$\begin{array}{llll}1 & (1) & a=b \wedge A[a / b] \wedge \Gamma & \text { Premise } \\ 1 & (2) & a=b & \wedge \mathrm{E}, 1 \\ 1 & (3) & A[a / b] & \wedge \mathrm{E}, 1 \\ 1 & (4) & A[b] & =\mathrm{E}, 2,3 \\ 1 & (5) & A[b] \wedge a=b \wedge A[a / b] \wedge \Gamma & \wedge \mathrm{I}, 1,4 \\ 1 & (6) & \Delta & \text { by (i) }\end{array}$

\subsubsection{Quarc to LK-Quarc}

1. Since in Quarc $a=a$ is introduced without relying on any previous steps, and given Definition 8, we need to show that the sequent $\Rightarrow a=a$ (with the left hand side empty) is derivable in LK-Quarc 2 . This is simple since $a=a \Rightarrow a=a$ is an initial sequent, the segment of a derivation corresponding to the rule $=\mathrm{I}$ is as follows:

$\frac{a=a \Rightarrow a=a}{\Rightarrow a=a}\left(=_{1}\right)$

2. The segment of a derivation corresponding to the rule $=\mathrm{E}$ is as follows:

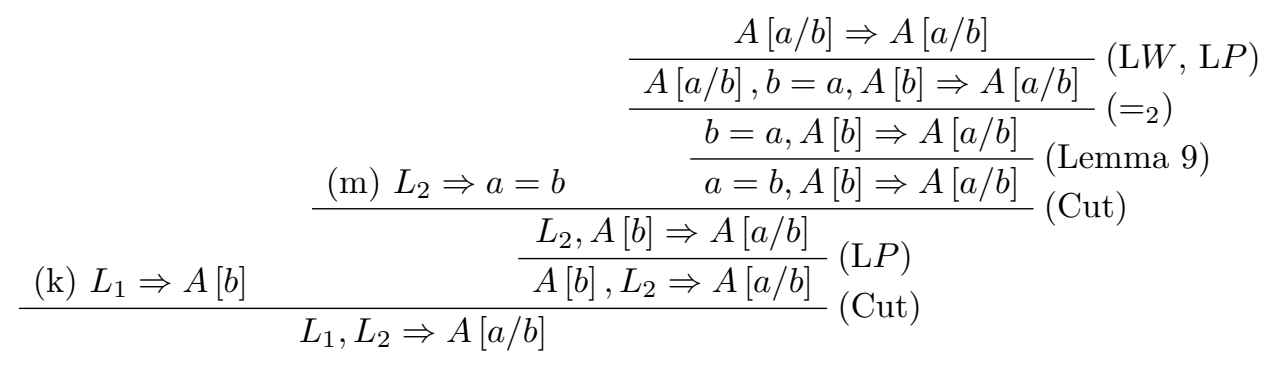

This concludes the proof of deductive equivalence of LK-Quarc 2 and $\mathrm{Quarc}_{2}$.

\subsection{Cut Elimination}

We prove the Cut elimination theorem for LK-Quarc 2 :

Theorem 6 For any sequent $S$, if $S$ is provable in LK-Quarc 2 , then it is provable in LK-Quarc 2 without using the cut rule. 
Proof. By expanding the proof for LK-Quarc $B$. Clearly, in both rules for identity all the formulas appearing in the lower sequent also appear in the upper sequent. Therefore, we only need to expand the proof for $\rho>2$.

\subsubsection{The Rule $\left(=_{1}\right)$}

The rule $={ }_{1}$ fits into the general proof for one-sequent derivations in the case $\rho>2$ (if $\rho=2$ then $={ }_{1}$ cannot be the last rule before the mix). Let only the right rank be greater than 1 . So, the application of the mix rule will be:

$$
\frac{\Gamma \Rightarrow \Theta \quad \frac{a=a, \Pi \Rightarrow \Delta}{\Pi \Rightarrow \Delta}\left({ }_{1}\right)}{\Gamma, \Pi^{*} \Rightarrow \Theta^{*}, \Delta} \text { (Mix) }
$$

If the mix formula is in $\Gamma$, then this transforms into a derivation with Mix eliminated altogether:

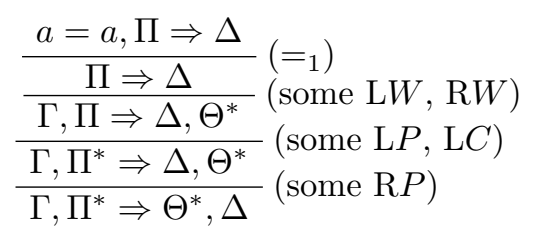

If the mix formula is not in $\Gamma$, then this transforms into :

$$
\begin{gathered}
\frac{\Gamma \Rightarrow \Theta \quad a=a, \Pi \Rightarrow \Delta}{\Gamma, a=a, \Pi^{*} \Rightarrow \Theta^{*}, \Delta} \\
\frac{\frac{a=a, \Pi^{*}, \Gamma \Rightarrow \Theta^{*}, \Delta}{(\text { Mix })}}{(\text { some L } P)}\left(={ }_{1}\right) \\
\frac{\Pi^{*}, \Gamma \Rightarrow \Theta^{*}, \Delta}{\Gamma, \Pi^{*} \Rightarrow \Theta^{*}, \Delta}(\text { some L } P)
\end{gathered}
$$

Since the right rank was reduced by 1 , while the left remains the same, the rank of the resulting mix rule is one less and, by inductive hypothesis, it can be eliminated. Similarly when the left rank, and both left and right rank, are greater than 1.

We need to examine the rule $=_{2}$ more closely, since it has two principal formulas, which also occur as the side formulas, and either of which could be the mix formula.

\subsubsection{The Rule $\left(=_{2}\right)$}

The case that needs to be examined here is when either $a=b$ or $A[a / b]$ is the mix formula. Assume it is $a=b$. The application of the mix rule then looks as follows:

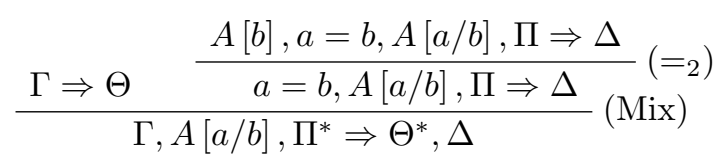


If the mix formula $a=b$ is in $\Gamma$, the derivation is transformed as follows:

$$
\begin{aligned}
& \frac{A[b], a=b, A[a / b], \Pi \Rightarrow \Delta}{a=b, A[a / b], \Pi \Rightarrow \Delta}\left(=_{2}\right) \\
& \frac{\frac{a}{a=b, \Pi, A[a / b] \Rightarrow \Delta}}{a=b, \Pi^{*}, A[a / b] \Rightarrow \Delta}(\text { some } \mathrm{L} P) \\
& \frac{\frac{\text { some } \mathrm{L} C)}{\Gamma, a=b, \Pi^{*}, A[a / b] \Rightarrow \Delta}}{\Gamma, \Pi^{*}, A[a / b] \Rightarrow \Delta}(\text { some } \mathrm{L} W) \\
& \frac{(\text { some } \mathrm{L} C)}{\Gamma, A[a / b], \Pi^{*} \Rightarrow \Theta^{*}, \Delta}
\end{aligned}
$$

Now suppose $a=b$ is not in $\Gamma$. Since $a=b$ is a basic formula, and by assumption the left rank is 1 , the sequent $\Gamma \Rightarrow \Theta$ is obtained by $\mathrm{R} W$ from $\Gamma \Rightarrow \Theta^{*}$. The derivation is then transformed as follows:

$$
\frac{\Gamma \Rightarrow \Theta^{*}}{\Gamma, A[a / b], \Pi^{*} \Rightarrow \Theta^{*}, \Delta}(\text { some } \mathrm{L} W, \mathrm{~L} P, \mathrm{R} W)
$$

Since $A[a / b]$ is likewise a basic formula, the same considerations will apply there. The remainder of the proof runs in parallel. This concludes the proof of the Theorem 6 .

\subsection{Subformula Property}

Here we can adopt a slightly weaker definition of subformula property, due to [13]:

Theorem 7 Any formula appearing in any cut-free proof of LK-Quarc ${ }_{2}$ is a subformula of some formula in its endsequent or a basic formula.

Proof. We only need to expand the proof of Theorem 4 with the cases for $={ }_{1}$ and $=_{2}$. However, these only remove basic formulas. Therefore, Theorem 7 holds.

Now, using this we can show consistency:

Corollary 2 LK-Quarc 2 is consistent.

Proof. From Theorem 7, by noting that basic formulas can only disappear from the left side of a sequent. Therefore, the empty sequent is not derivable.

\subsection{Conservativity}

Theorem 8 LK-Quarc 2 is conservative expansion of LK-Quarc ${ }_{B}$. Namely, if $\Gamma \Rightarrow \Delta$ is derivable in LK-Quarc 2 , and $\Gamma$ and $\Delta$ contain no identity, then $\Gamma \Rightarrow \Delta$ is derivable in $\mathrm{LK}$-Quarc ${ }_{B}$. 
Proof. Assume $\Gamma \Rightarrow \Delta$ is derivable in LK-Quarc 2 , and consider a cut-free derivation of $\Gamma \Rightarrow \Delta$. Moreover, assume $\Gamma$ and $\Delta$ contain no identity. By weak subformula property, it follows that

Corollary 3 Any formula in the derivation of $\Gamma \Rightarrow \Delta$ that contains identity is a basic formula.

Moreover, it follows that

Corollary 4 No formula containing identity occurs on the right side of any sequent in the derivation.

Furthermore, given that the rule $=_{2}$ can never reduce the number of formulas containing identity below 1 , and that the rule $={ }_{1}$ can only reduce the number of such formulas below 1 if they are of the form $a=a$, it follows that

Corollary 5 Any identity formula in the derivation of $\Gamma \Rightarrow \Delta$ is of the form $a=a$.

Take a (cut-free) derivation of $\Gamma \Rightarrow \Delta$. It is then transformed in two step.

First step. Any occurence of the rule $=_{2}$, given Corollary 5, is of the form:

$$
\frac{A[a], a=a, A[a / a], \Gamma^{\prime} \Rightarrow \Delta^{\prime}}{a=a, A[a / a], \Gamma^{\prime} \Rightarrow \Delta^{\prime}}\left(={ }_{2}\right)
$$

Since $A[a]$ and $A[a / a]$ are the same formula, this is transformed into

$$
\frac{A[a], a=a, A[a / a], \Gamma^{\prime} \Rightarrow \Delta^{\prime}}{a=a, A[a / a], \Gamma^{\prime} \Rightarrow \Delta^{\prime}}(\mathrm{L} C)
$$

Second step. Any occurrence of the rule $\mathbf{L} C$, where $a=a$ is the principal formula,

$$
\frac{a=a, a=a, \Gamma \Rightarrow \Delta}{a=a, \Gamma \Rightarrow \Delta}(\mathrm{L} C)
$$

Is transformed into an occurrence of the rule $={ }_{1}$ :

$$
\frac{a=a, a=a, \Gamma \Rightarrow \Delta}{a=a, \Gamma \Rightarrow \Delta}\left(={ }_{1}\right)
$$

Observation 1 Obviously, both these transformations yield correct derivations. After completing both, the rule $=_{2}$ does not occur, and the formula $a=a$ is the principal formula of either the rule $=_{1}$ or $\mathbf{L} W$ (since by Corollary 4 it cannot occur in an initial sequent).

We now proceed to prove the above theorem by proving the following lemma:

Lemma 10 Any occurence of the formula $a=a$ in the derivation of $\Gamma \Rightarrow \Delta$ can be eliminated. 
Proof. Given Observation 1, every formula of the form $a=a$ will form a chain of sequents, such that the first sequent of the chain is the lower sequent of a $\mathbf{L} W$ rule with $a=a$ as a principal formula, and the last sequent of the chain the upper sequent of $\mathrm{a}={ }_{1}$ rule with $a=a$ as a principal formula. Let the length of such a chain be the number of sequents in the chain.

We only need to show that such a chain ending with the topmost leftmost occurrence of $={ }_{1}$ can be eliminated. The proof is by induction on the length of the chain.

Basic step. The shortest chain has length 1, and is of the following form:

$$
\frac{\frac{\Gamma^{\prime} \Rightarrow \Delta^{\prime}}{a=a, \Gamma^{\prime} \Rightarrow \Delta^{\prime}}}{\Gamma^{\prime} \Rightarrow \Delta^{\prime}}\left({ }_{1}\right)
$$

This is transformed into the derivation of the upper sequent $\Gamma^{\prime} \Rightarrow \Delta^{\prime}$, which by Corollary 4 does not contain the formula $a=a$.

Inductive step. Let the end of a chain be (where Inf is any derivation rule)

$$
\frac{\frac{a=a, \Gamma^{\prime \prime} \Rightarrow \Delta^{\prime \prime}}{a=a, \Gamma^{\prime} \Rightarrow \Delta^{\prime}}}{\Gamma^{\prime} \Rightarrow \Delta^{\prime}}\left({ }_{1}\right)
$$

Since $a=a$ is not principal in Inf, this can be transformed into

$$
\frac{a=a, \Gamma^{\prime \prime} \Rightarrow \Delta^{\prime \prime}}{\frac{\Gamma^{\prime \prime} \Rightarrow \Delta^{\prime \prime}}{\Gamma^{\prime} \Rightarrow \Delta^{\prime}}(\mathrm{Inf})}\left(={ }_{1}\right)
$$

Where the length of the chain is reduced by one. Similarly for the two-sequent rules. This concludes the proof of Lemma 10.

By Corollary 5 and Lemma 10 if follows that the derivation transformed in this manner contains no identity. Moreover, it contains no rule $=_{2}$ (Observation 1) nor $=_{1}$ (Lemma 10). Therefore, it is a derivation of LK-Quarc ${ }_{B}$. This concludes the proof of Theorem 8 .

\section{$7 \quad$ Particular Import in LK-Quarc ${ }_{B}$}

Having proven the Cut elimination theorem, we now proceed to use it in further considerations. The first application will be to demonstrate that particular import is not derivable in LK-Quarc ${ }_{B}$ and therefore, given deductive equivalence result of Theorem 2, it is likewise not derivable in $\mathrm{Quarc}_{B}$.

As we have seen in a simplified version in Example 2 in Section 2, DeMorgan laws hold in Quarc $_{B}$ (and consequently in Quarc as well).

But, we will demonstrate that 
Theorem 9 The sequent $\forall M P \Rightarrow \neg \forall M \neg P$, and therefore particular import, is not derivable in $\mathrm{LK}$-Quarc $B$.

Proof. Suppose there is a cut-free proof of $\forall M P \Rightarrow \neg \forall M \neg P$ in LK-Quarc ${ }_{B}$. Then, there is also a cut-free proof of $\forall M \neg P, \forall M P \Rightarrow$. This sequent may undergo any number of applications of $\mathrm{L} W, \mathrm{~L} C$ and $\mathrm{L} P$, resulting in a sequent $(\forall M \sim P)_{1}, \ldots,(\forall M \sim P)_{n} \Rightarrow$, where $\sim$ in each of the formulas $1, \ldots, n$ stands either for negation or an empty string of symbols. Assume that the rules in the proof are applied in the following order: the left structural rules are applied below any application of $\mathrm{L} \forall$, and all applications of $\mathrm{L} \forall$ are below any application of $\mathrm{R} W$.

By observing the rule $\mathrm{L} \forall$ we can see that the top right sequent just above the topmost application of $\mathrm{L} \forall$ will be $\Rightarrow(a M)_{1}, \ldots,(a M)_{n}$. Above this sequent $\mathrm{R} W$ can be applied $n-1$ times, resulting in a top right sequent of the proof being $\Rightarrow a M$.

It is clear that any other order of application of rules will result in the same top right sequent - every lower application of $\mathrm{L} \forall$ on a formula $\forall M \sim P$ will result in an additional $a M$ in the right sequent, and no application of $\mathrm{R} W$ on it will reduce the number of formulas $a M$ in it below one. But, $\Rightarrow a M$ is not an initial sequent (and neither is any other sequent of the same branch). Therefore, there is no cut-free proof of $\forall M \neg P, \forall M P \Rightarrow$ in LK-Quarc ${ }_{B}$, and so no cut-free proof of $\forall M P \Rightarrow \neg \forall M \neg P$. Given the cut elimination theorem, this means there is no proof of $\forall M P \Rightarrow \neg \forall M \neg P$ in LK-Quarc $B$.

This concludes the proof of Theorem 9. This is the last obstacle to expanding LK-Quarc $B$ into a sequent calculus deductively equivalent with full Quarc. In the following subsection, we will see how to expand LK-Quarc $B$ with a rule that will give the resulting system equivalence with Quarc which includes Instantiation $\left(\right.$ Quarc $\left._{3}\right)$.

\subsection{Instantiation Rule}

To expand LK-Quarc ${ }_{B}$ into LK-Quarc 3 , we add the rule for Instantiation:

$$
\frac{t M, \Gamma \Rightarrow \Delta}{\Gamma \Rightarrow \Delta}(\mathrm{Ins})^{*}
$$

* - where neither $\Gamma$ nor $\Delta$ contain the singular argument $t$.

This rule allows for the derivation of a particular sentence from a corresponding sentence governed by the universal quantified argument: 
Theorem $10 A[\forall S] \Rightarrow A[\exists S / \forall S]$

Proof.

$$
\begin{gathered}
\frac{a S \Rightarrow a S}{\frac{A[\forall S], a S \Rightarrow a S}{a S, A[\forall S] \Rightarrow a S}(\mathrm{~L} P)} \quad \frac{}{A[\forall S], a S \Rightarrow A[a / \forall S]} \text { (Lemma 4) } \\
\frac{a S P, A[\forall S] \Rightarrow A[\exists S / \forall S]}{A[\forall S] \Rightarrow A[\exists S / \forall S]} \text { (Ins) }
\end{gathered}
$$

Moreover, it allows for the derivation of a theorem

Theorem $11 \Rightarrow(\exists S) S$

Proof.

$$
\frac{a S \Rightarrow a S \quad a S \Rightarrow a S}{\frac{a S \Rightarrow(\exists S) S}{\Rightarrow(\exists S) S} \text { (Ins) }}
$$

However, this rule will not allow the derivation of the problematic sequent ' $\Rightarrow a M$ ' from the proof of Theorem 9 , since the following is not a permissible application of this rule:

$$
\frac{a M \Rightarrow a M}{\Rightarrow a M} *(\mathrm{Ins})
$$

So, this sequent calculus is, at least prima facie, powerful enough, without being too powerful. We now formalize this result.

\subsection{Deductive Equivalence}

Theorem 12 Quarc $_{3}$ and LK-Quarc 3 are deductively equivalent.

Proof. In addition to the proof of Lemma 2, we need to show that

5. (Ins) Assume (i) $t S \wedge \Gamma \vdash \Delta$ and (ii) $\Gamma$ and $\Delta$ do not contain $t$. Now assume (1) $\Gamma$. We need to derive $\Delta$. 


$\begin{array}{rlll}1 & (1) & \Gamma & \text { Premise } \\ 2 & (2) & (t) S & \text { Premise } \\ & (3) & (\forall S) S & \text { UI, } 2,2 \\ 4 & (4) & (t) S & \text { Premise } \\ 5 & (5) & (t) S & \text { Premise } \\ 1,4 & (6) & (t) S \wedge \Gamma & \wedge \mathrm{I}, 1,4 \\ 1,4 & (7) & \Delta & \text { by (i) } \\ 1 & (8) & \Delta & \text { Ins, } 3,4,5,7 \text { given (ii) }\end{array}$

In addition to the proof of Lemma 3 we need to construct a corresponding segment of a derivation for the Instantiation rule of $\mathrm{Quarc}_{3}$.

8. (Ins) The Instantiation rule has the following form:

$\begin{array}{rlll}L_{1} & (\mathrm{i}) & A[q P] & \\ j & (\mathrm{j}) & (t) P & \text { Premise } \\ k & (\mathrm{k}) & A[t / q P] & \text { Premise } \\ L_{2} & (\mathrm{l}) & B & \\ L_{1} \cup L_{2}-\{j, k\} & (\mathrm{m}) & B & \text { Ins, i, j, k, l }\end{array}$

where $\mathrm{L}_{1}, B$ and $A[q P]$ do not contain the singular argument $t$, and in $L_{2}$ the only occurrences of $t$ are in $(\mathrm{j})$ and $(\mathrm{k})$.

Since we have already demonstrated Lemma 3 for the particular quantifier, we need to concern ourselves only with the cases where $q$ stands for the universal quantifier $\forall$. The corresponding segment of that derivation is as follows (let $L_{2} *$ be the list $L_{2}$ with (j) and (k) omitted - it thus contains no singular argument $t$ ):

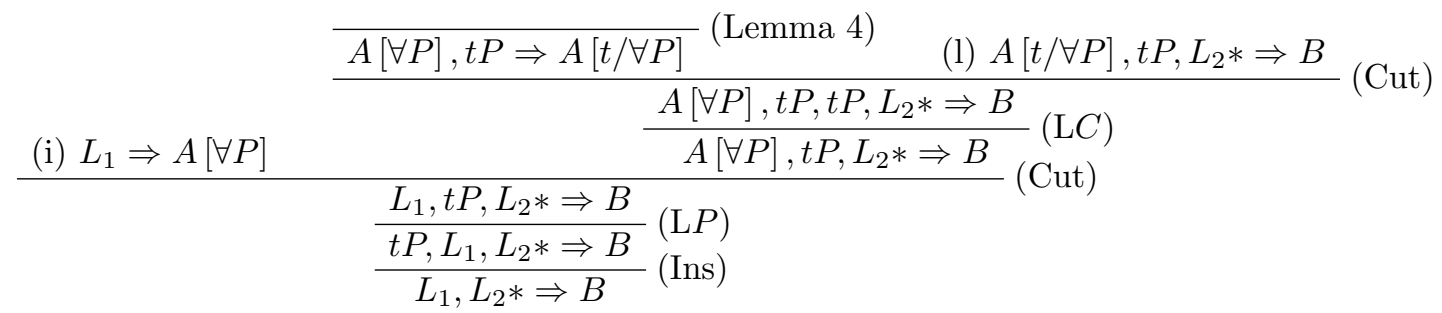

Since neither $L_{1}, L_{2} *$ nor $B$ contain the singular argument $t$, this is an appropriate use of the Ins rule of LK-Quarc 3 . Of course, for this segment to have the appropriate form of using all the steps listed in the justification in Quarc, the segment above the application of Lemma 4 should have the following form: 


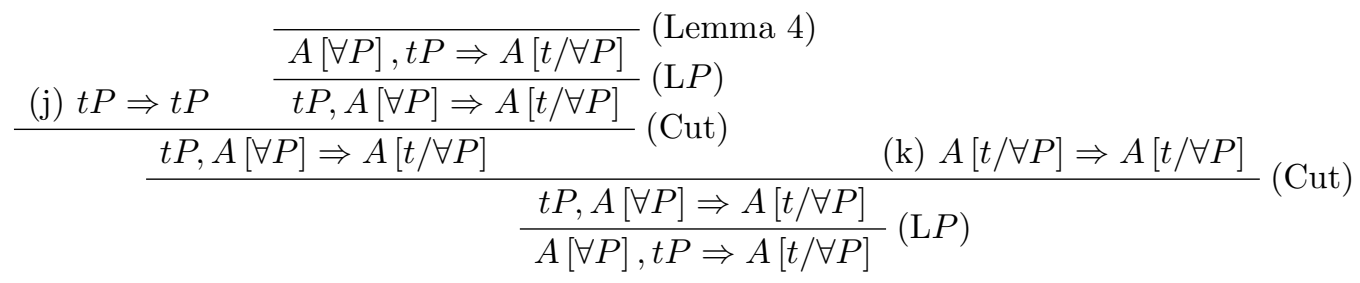

However, since steps $(\mathrm{j})$ and $(\mathrm{k})$ are always premises, the above segment will suffice on its own.

\subsection{Cut Elimination}

Here we need to check only the cases where $\rho>2$. Let the right rank be greater than 1 . So, the application of the mix rule will be:

$$
\frac{\Gamma \Rightarrow \Theta \quad \frac{t M, \Pi \Rightarrow \Delta}{\Pi \Rightarrow \Delta} \text { (Ins) }}{\Gamma, \Pi^{*} \Rightarrow \Theta^{*}, \Delta} \text { (Mix) }
$$

(suppose the mix formula $\mathrm{A}$ is not in $\Gamma$ ). Then this transforms into:

$$
\begin{gathered}
\frac{\Gamma \Rightarrow \Theta \quad t M, \Pi \Rightarrow \Delta}{\Gamma, t M, \Pi^{*} \Rightarrow \Theta^{*}, \Delta}(\text { Mix) } \\
\frac{t M, \Pi^{*}, \Gamma \Rightarrow \Theta^{*}, \Delta}{\Pi^{*}, \Gamma \Rightarrow \Theta^{*}, \Delta} \text { (Ins) } \mathrm{L} P \text { ) } \\
\frac{\Gamma, \Pi^{*} \Rightarrow \Theta^{*}, \Delta}{\Gamma \text { (some LP) }} \text { (s) }
\end{gathered}
$$

Since the right rank was reduced by 1 , while the left remains the same, the rank of the resulting mix rule is one less and, by inductive hypothesis, it can be eliminated. Similarly when the left rank is greater than 1.

\subsection{Subformula Property}

The reasoning here runs in parallel to Theorem 7:

Theorem 13 Any formula appearing in any cut-free proof of LK-Quarc ${ }_{3}$ is a subformula of some formula in its endsequent or a basic formula.

Proof. We only need to expand the proof of Theorem 4 with the case for Ins. However, it only removes basic formulas. Therefore, Theorem 13 holds.

And consistency follows:

Corollary 6 LK-Quarc 3 is consistent.

Proof. Same as Corollary 2. 


\subsection{Conservativity of LK-Quarc over LK-Quarc 3}

Given the cut elimination property and the subformula property of LK-Quarc 3 , it follows that

Theorem 14 LK-Quarc is a conservative expansion of LK-Quarc 3 . Namely, if $\Gamma \Rightarrow \Delta$ is derivable in LK-Quarc, and $\Gamma$ and $\Delta$ contain no identity, then $\Gamma \Rightarrow \Delta$ is derivable in $\mathrm{LK}-\mathrm{Quarc}_{3}$.

Proof. Same as Theorem 8.

From this it follows as a corollary that (note that Quarc 3 is in fact Ben-Yami's original system from [3]):

Corollary 7 Quarc $_{3}$ is complete. Namely, if $\Gamma \vDash \Delta$ in Quarc $_{3}$ then $\Gamma \vdash \Delta$ in Quarc $_{3}$.

Proof. Assume $\Gamma \vDash \Delta$ in Quarc $_{3}$. Therefore $\Gamma$ and $\Delta$ do not contain identity. Given that, and since Quarc 3 and Quarc assign the same values to all formulas not containing identity, it follows that also $\Gamma \vDash \Delta$ in Quarc. Since Quarc is complete, it follows that $\Gamma \vdash \Delta$ in Quarc. Given Deductive equivalence (Theorem 4), $\Gamma \Rightarrow \Delta$ in LK-Quarc. Now, given Theorem 14 (and since $\Gamma$ and $\Delta$ do not contain identity), $\Gamma \Rightarrow \Delta$ in LK-Quarc 3 . Finally, given Deductive equivalence (Theorem 4), $\Gamma \vdash \Delta$ in Quarc $_{3}$.

Therefore, using the analysis of this paper, we are also able to demonstrate completeness of Ben-Yami's original system from the completeness of the system with identity from [5].

\section{Concluding remarks}

In this paper we have provided a concise proof-theoretic study of Quarc within LK-systems. An obvious next step would naturally be completeness which follows from the deductive equivalences [5]. Moreover, there is also a more direct way of establishing this important theorem, by adopting a proof of completeness that is typical for sequent calculus [14], [6].

Possible topics for further research include an interpolation theorem for the various LK-Quarc systems; thereby we could also examine Beth's definability theorem. On a more philosophical side Quarc enriched by modalities - as suggested by [3] - and correspondingly with its expansion of expressive power, provides ample opportunity for exploration. 


\section{References}

[1] M. Baaz, A. Leitsch, Methods of Cut-Elimination, Springer, Trends in Logicseries 34, 2011.

[2] E. Bencivenga, Free Logics in: D. Gabbay, F. Guenthner (eds), Handbook of Philosophical Logic, 2nd Edition, Vol. 5, Springer Science+Media Dordrecht, pp.147-196, 2002.

[3] H. Ben-Yami, The Quantified Argument Calculus, The Review of Symbolic Logic, pp. 120-146, 2014.

[4] H. Ben-Yami, Logic and Natural Language, Ashgate, 2004.

[5] H. Ben-Yami, E. Pavlovic, Completeness of the Quantified Argument Calculus, manuscript.

[6] S. Buss An Introduction to Proof Theory, in: S. Buss (ed), Handbook of Proof Theory, Elsevier, pp.1-78, 1998.

[7] G. Gentzen, The Collected Papers of Gerhard Gentzen, ed. M. Szabo, NorthHolland, pp. 68-131, 1969.

[8] N. Gratzl, A Sequent Calculus for a Negative Free Logic, Studia Logica 96, pp.331-348, 2010.

[9] S.C. Kleene, Introduction to Metamathematics (13 ${ }^{\text {th }}$ edition), WoltersNoordhoff Publishing, 2000.

[10] K. Lambert, Free Logics: Their Foundations, Character, and Some Applications Thereof, Academia Verlag, 1997.

[11] K. Lambert, Free Logics in: L. Goble (ed), The Blackwell Guide to Philosophical Logic, Blackwell Publishers, pp.258-279, 2001.

[12] R. Lanzet, H. Ben-Yami, Logical Inquiries into a New Formal System with Plural Reference, First-order logic revisited, Logische Philosophie series 12, pp. 173-223, 2006.

[13] S. Negri, J. von Plato, Structural Proof Theory, Cambridge: Cambridge University Press, 2001.

[14] K. Schütte, Beweistheorie, Springer, 1960.

[15] G. Takeuti, Proof Theory (2 ${ }^{\text {nd }}$ edition), North-Holland, 1987. 
Edi Pavlović

Unioninkatu 40,

00170 Helsinki

Finland

Edi.Pavlovic@helsinki.fi

Norbert Gratzl

Ludwig-Maximilians-Universität München

Fakultät für Philosophie, Wissenschaftstheorie und Religionswissenschaft

Munich Center for Mathematical Philosophy (MCMP)

Geschwister-Scholl-Platz 1

D-80539 München

N.Gratzl@lmu.de 